\title{
C-Terminal Domain Swapping of SSB Changes the Size of the ssDNA Binding Site
}

\author{
Yen-Hua Huang ${ }^{1}$ and Cheng-Yang Huang ${ }^{1,2}$ \\ ${ }^{1}$ School of Biomedical Sciences, Chung Shan Medical University, No.110, Sec.1, Chien-Kuo N. Rd., Taichung City, Taiwan \\ ${ }^{2}$ Department of Medical Research, Chung Shan Medical University Hospital, No.110, Sec.1, Chien-Kuo N. Rd., Taichung City, Taiwan \\ Correspondence should be addressed to Cheng-Yang Huang; cyhuang@csmu.edu.tw
}

Received 26 May 2014; Accepted 9 July 2014; Published 4 August 2014

Academic Editor: Huangen Ding

Copyright (c) 2014 Y.-H. Huang and C.-Y. Huang. This is an open access article distributed under the Creative Commons Attribution License, which permits unrestricted use, distribution, and reproduction in any medium, provided the original work is properly cited.

\begin{abstract}
Single-stranded DNA-binding protein (SSB) plays an important role in DNA metabolism, including DNA replication, repair, and recombination, and is therefore essential for cell survival. Bacterial SSB consists of an N-terminal ssDNA-binding/oligomerization domain and a flexible C-terminal protein-protein interaction domain. We characterized the ssDNA-binding properties of Klebsiella pneumoniae SSB (KpSSB), Salmonella enterica Serovar Typhimurium LT2 SSB (StSSB), Pseudomonas aeruginosa PAO1 SSB (PaSSB), and two chimeric KpSSB proteins, namely, KpSSBnStSSBc and KpSSBnPaSSBc. The C-terminal domain of StSSB or PaSSB was exchanged with that of KpSSB through protein chimeragenesis. By using the electrophoretic mobility shift assay, we characterized the stoichiometry of KpSSB, StSSB, PaSSB, KpSSBnStSSBc, and KpSSBnPaSSBc, complexed with a series of ssDNA homopolymers. The binding site sizes were determined to be $26 \pm 2,21 \pm 2,29 \pm 2,21 \pm 2$, and $29 \pm 2$ nucleotides (nt), respectively. Comparison of the binding site sizes of KpSSB, KpSSBnStSSBc, and KpSSBnPaSSBc showed that the C-terminal domain swapping of SSB changes the size of the binding site. Our observations suggest that not only the conserved $\mathrm{N}$-terminal domain but also the C-terminal domain of SSB is an important determinant for ssDNA binding.
\end{abstract}

\section{Introduction}

Single-stranded DNA-binding protein (SSB) specifically binds to single-stranded DNA (ssDNA) and is known to have important functions in the DNA metabolic processes, such as DNA replication, repair, and recombination of both prokaryotes and eukaryotes [1-4]. During these reactions, SSB binds to and protects susceptible ssDNA from nucleolytic digestion and chemical attacks and also prevents secondary structure formation [5]. Many but not all bacterial and human mitochondrial SSBs are active as homotetramers [57], in which four oligonucleotide/oligosaccharide-binding folds (OB folds) form a DNA-binding domain [8-12]. However, SSB from the bacterial phylum Deinococcus-Thermus functions as a homodimer, in which each monomer contains two OB folds linked by a conserved spacer sequence [13-20]. SSB from Sulfolobus solfataricus is a monomer that includes one OB fold, which differentiates SSB from the bacterial form, and is likely to be a more ancestral "simple" SSB [21-25]. The
DdrB protein from Deinococcus radiodurans is an alternative SSB and functions as a pentamer [26]. Recent studies found that a distinct SSB from hyperthermophilic Crenarchaea, termed ThermoDBP, has ssDNA-binding domains that are markedly different from the classical OB folds of bacterial SSB $[27,28]$.

Bacterial SSBs consist of two domains, namely, an Nterminal ssDNA-binding/oligomerization domain and a flexible C-terminal protein-protein interaction domain without a defined tertiary structure [3,29]. Tyrosine phosphorylation of SSB increases binding to ssDNA by almost 200 -fold in vitro $[30,31]$. The $\mathrm{N}$-terminal domain is separated from the highly conserved acidic tail of the last $10 \mathrm{C}$-terminal amino acid residues of SSB by a long proline- or glycine-rich hinge $[3,32]$. SSB interacts with other auxiliary proteins that are essential for cell survival [33]. The C-terminal acidic tail of SSB, such as "DDDIPF", has been shown to bind to more than a dozen different proteins and the activity of some of these proteins is stimulated by their interactions with ssDNA-bound SSB [3]. 
The binding of SSB to ssDNA makes the glycine-rich region more easily accessible to other proteins such as proteases and DNA polymerase III $[33,34]$. The C-terminus in SSB can also interact with the $\mathrm{OB}$ fold and regulate the ssDNA-binding activity of SSB itself $[35,36]$.

Studies on SSB from different organisms have grown rapidly during the past few years and knowledge on how SSBs interact with ssDNA has increased [22, 32, 37-46]. The most thoroughly studied SSB is that of Escherichia coli (EcSSB), which binds cooperatively to ssDNA [47]. The estimated binding site size of EcSSB is dependent on the salt concentration in fluorescence titrations with poly(dT) [47]. EcSSB mainly binds to 35- and 65-nucleotide- (nt) long ssDNA via the $(\mathrm{SSB})_{35^{-}}$and $(\mathrm{SSB})_{65}$-binding modes, respectively. In the $(\mathrm{SSB})_{35}$-binding mode, two subunits of the EcSSB tetramer interact with ssDNA, whereas in the $(\mathrm{SSB})_{65^{-}}$ binding mode all four subunits participate in ssDNA binding. These different binding modes may be required during different stages of DNA metabolism for the in vivo function of SSB [48-50]. Although SSB binds to ssDNA via the highly conserved ssDNA-binding domain, the reason that the binding site sizes of SSBs from different organisms differ remains unclear. For example, differences are found among the binding site sizes of Methanococcus jannaschii SSB [51], the Gonococcal Genetic Island-encoded SSB from Neisseria gonorhoeae [39], the thermostable Thermotoga maritima and Thermotoga neapolitana SSBs [32], and the psychrophilic bacterial SSBs [37]. In addition, the $(\mathrm{SSB})_{35^{-}}$and $(\mathrm{SSB})_{65}$-binding modes are not found in some SSBs [32, 39, 42].

Previously, we have examined the electrophoretic mobility shift patterns of a His-tagged Klebsiella pneumoniae SSB (KpSSB) [40], a His-tagged Salmonella enterica serovar Typhimurium LT2 SSB (StSSB) [43], and a His-tagged Pseudomonas aeruginosa PAO1 SSB (PaSSB) [42] bound to different lengths of ssDNA. We also determined their corresponding binding site sizes, that is, 26, 22, and 29 nt per tetramer, respectively. The electrophoretic mobility shift assay (EMSA) is a well-established approach in studies of molecular biology [52], and the use of radioactive tracer in this assay allows visualization of the actual formation of the distinct protein-DNA complex(es)[53]. The expected result of EMSA is that when the length of the nucleotides is sufficient for the binding of two or more SSB molecules, the electrophoretic mobility of the higher SSB oligomer complex will be lower than that of the smaller SSB oligomer complex [52, 54]. Recent studies on SSB binding also reveal that determination of the ssDNAbinding site size by using EMSA is significantly consistent with that of the cocrystal structure of SSB with ssDNA [27].

KpSSB, StSSB, and PaSSB are similar proteins whose $\mathrm{N}$-terminal ssDNA-binding domains are almost identical, except for different ssDNA-binding site sizes [40, 42, 43]. Thus, we should assess whether the glycine-rich hinge, which is not conserved among SSBs, is involved in the determination of the binding site size of SSB. In this study, we swapped the C-terminal domains of StSSB and PaSSB into that of KpSSB through protein chimeragenesis. Chimeras are proteins that contain segments from two or more different parent proteins and serve as valuable tools to understand enzyme mechanism and protein function [55]. The EMSA behavior (patterns) of the resultant chimeric proteins, namely, $\mathrm{KpSSBnStSSBc}$ and $\mathrm{KpSSBnPaSSBc}$, was characterized and compared with untagged KpSSB, StSSB, and PaSSB (Figure 1). On the basis of the chimeragenesis results, the flexible C-terminal domain of SSB was found to be involved in determining the ssDNA-binding site sizes.

\section{Materials and Methods}

2.1. Materials. All restriction enzymes and DNA-modifying enzymes were purchased from New England Biolabs (Ipswich, MA, USA) unless explicitly stated otherwise. All chemicals were purchased from Sigma-Aldrich (St. Louis, MO, USA) unless explicitly stated otherwise. The E. coli strains TOP10F' (Invitrogen, USA) and BL21(DE3)pLysS (Novagen, UK) were used for genetic construction and protein expression, respectively.

\subsection{Construction of Plasmids for KpSSB, StSSB, and PaSSB} Expression. The KpSSB [40], StSSB [43], and PaSSB [42] expression plasmids were constructed by the protocols described previously, with minor modification, to avoid having a His tag fused with the gene product. A fragment containing the coding sequence of KpSSB (KPN04446), StSSB (STM4256), and PaSSB (PA4232) (with the stop codon) was directly amplified by PCR by using the genomic DNA of K. pneumoniae subsp. pneumoniae MGH 78578, S. enterica serovar Typhimurium LT2, or $P$. aeruginosa PAO1 (Primers 1 to 6 , resp.). During the process, NdeI and XhoI restriction sites were introduced at the $5^{\prime}$-end and the $3^{\prime}$-end of these genes, after which they were ligated into the pET21b vector (Novagen Inc., Madison, WI, USA) for protein expression in E. coli BL21. The expected gene product expressed by these plasmids does not contain any artificial residue, including a His tag. Primers used for construction of these plasmids are summarized in Table 1.

2.3. Construction of Plasmids for KpSSBnStSSBc and KpSSBn$\mathrm{PaSSB}$ C Expression through Protein Chimeragenesis. To investigate the effect of the C-terminal domain of SSB on the size of the ssDNA-binding site, the C-terminal domain of KpSSB was replaced by that of StSSB and PaSSB. pET21bKpSSB (Primers 7 and 8), pET21b-StSSB (Primers 9 and 10 ), and pET21b-PaSSB (Primers 11 and 12) vectors were mutated to create a desired SacI site and to obtain the vectors for expression of the chimeric proteins KpSSBnStSSBc and KpSSBnPaSSBc. The D91E/Q92L-engineered pET21b-KpSSB vector, the D91E/Q92L-engineered pET21bStSSB vector, and the G90E/Q91L-engineered pET21b-PaSSB vector were cut at NdeI and SacI sites. Subsequently, the KpSSBn, StSSBc-pET21b, and PaSSBc-pET21b fragments were purified. KpSSBn was ligated with StSSBc-pET21b and PaSSBc-pET21b fragments to generate the engineered pET21b-KpSSBnStSSBc and pET21b-KpSSBnPaSSBc vectors. To avoid artificial residues, positions 91 and 92 of the two plasmids were mutated back (Primers 13 to 16) to obtain pET21b-KpSSBnStSSBc and pET21b-KpSSBnPaSSBc vectors. 

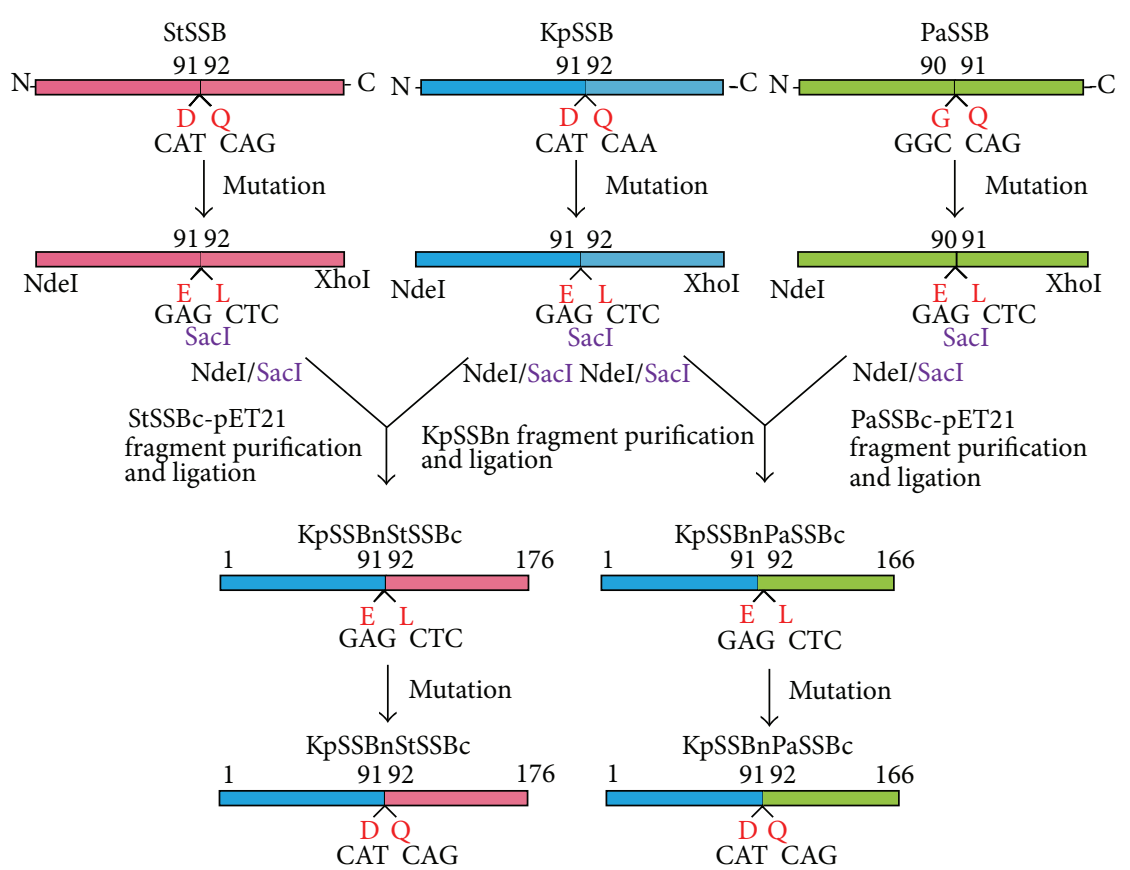

FIGURE 1: Construction of plasmids for expression of the chimeric KpSSBnStSSBc and KpSSBnPaSSBc proteins. To investigate the effect of the C-terminal domain of SSB on the size of the ssDNA-binding site, the C-terminal domain of KpSSB was replaced by that of StSSB and PaSSB. pET21b-KpSSB (Primers 7 and 8), pET21b-StSSB (Primers 9 and 10), and pET21b-PaSSB (Primers 11 and 12) vectors were mutated to create a desired SacI site and to obtain the vectors for expression of the chimeric proteins KpSSBnStSSBc and KpSSBnPaSSBc. The D91E/Q92Lengineered pET21b-KpSSB vector, the D91E/Q92L-engineered pET21b-StSSB vector, and the G90E/Q91L-engineered pET21b-PaSSB vector were cut at NdeI and SacI sites. Subsequently, the KpSSBn, StSSBc-pET21b, and PaSSBc-pET21b fragments were purified. KpSSBn was ligated with StSSBc-pET21b and PaSSBc-pET21b fragments to generate the engineered pET21b-KpSSBnStSSBc and pET21b-KpSSBnPaSSBc vectors. To avoid artificial residues, positions 91 and 92 of the two plasmids were mutated back (Primers 13 to 16) to obtain pET21b-KpSSBnStSSBc and pET21b-KpSSBnPaSSBc vectors. Thus, pET21b-KpSSBnStSSBc and pET21b-KpSSBnPaSSBc will express KpSSB1-91 fused StSSB92-176 and PaSSB91-165, respectively. Note that KpSSBnPaSSBc will have 166 amino acid residues.

Thus, pET21b-KpSSBnStSSBc and pET21b-KpSSBnPaSSBc will express KpSSB1-91 fused StSSB92-176 and PaSSB91-165, respectively. Note that KpSSBnPaSSBc will have 166 amino acid residues. Plasmids were verified by DNA sequencing. Underlined nucleotides indicate the designated site for mutation or the restriction site (Table 1).

2.4. Protein Expression and Purification. The recombinant SSBs were expressed using the protocol described previously $[9,40,42,43,56-60]$. Purification of these recombinant SSBs was carried out as described previously with the following modifications [61, 62]. Briefly, E. coli BL21(DE3) cells were individually transformed with the expression vector and grown to $\mathrm{OD}_{600}$ of 0.9 at $37^{\circ} \mathrm{C}$ in Luria-Bertani medium containing $250 \mu \mathrm{g} / \mathrm{mL}$ ampicillin with rapid shaking. Overexpression of the expression plasmids was induced by incubating with $1 \mathrm{mM}$ isopropyl thiogalactoside (IPTG) for $3 \mathrm{~h}$ at $37^{\circ} \mathrm{C}$. The cells overexpressing the protein were chilled on ice, harvested by centrifugation, resuspended in Buffer A $(20 \mathrm{mM}$ Tris- $\mathrm{HCl}, 5 \mathrm{mM}$ imidazole, and $0.2 \mathrm{M}$ ammonium sulfate, $\mathrm{pH}$ 7.9), and disrupted by sonication with ice cooling. The protein solution $(50 \mathrm{~mL})$ was precipitated from the supernatant of the cell lysate by incubation with $0.27 \mathrm{~g} / \mathrm{mL}$ of ammonium sulfate for $30 \mathrm{~min}$ and centrifugation at $20000 \mathrm{~g}$ for $10 \mathrm{~min}$. The pellets were washed twice with $2.0 \mathrm{~mL}$ of Buffer B $(20 \mathrm{mM}$
Tris- $\mathrm{HCl}, 5 \mathrm{mM}$ imidazole, and $1.2 \mathrm{M}$ ammonium sulfate, $\mathrm{pH}$ 7.9). After dialysis against Buffer $\mathrm{C}(20 \mathrm{mM}$ Tris- $\mathrm{HCl}$, $5 \mathrm{mM}$ imidazole, $1 \mathrm{mM}$ EDTA, and $100 \mathrm{mM} \mathrm{NaCl}, \mathrm{pH} 7.9$ ), the protein solution applied to the Q column (GE Healthcare BioSciences, Piscataway, NJ, USA) was eluted with a linear $\mathrm{NaCl}$ gradient from 0.1 to $0.6 \mathrm{M}$ with Buffer $\mathrm{C}$ using the AKTAFPLC system (GE Healthcare Bio-Sciences, Piscataway, NJ, USA). The peak fractions with the ssDNA-binding activity were collected and dialyzed against Buffer $\mathrm{D}(20 \mathrm{mM}$ potassium phosphate, $1 \mathrm{mM}$ EDTA, and $100 \mathrm{mM} \mathrm{NaCl}, \mathrm{pH} 7.0$ ). The protein solution was then applied to the Heparin HP column (GE Healthcare Bio-Sciences, Piscataway, NJ, USA) and eluted with a linear $\mathrm{NaCl}$ gradient from 0.1 to $1.0 \mathrm{M}$ with Buffer D. The peak fractions from this chromatographic step with the ssDNA-binding activity were collected and concentrated, and the purity of these SSBs was checked by Coomassie-stained SDS-PAGE (Mini-PROTEAN Tetra System, Bio-Rad, CA, USA; Figure 3).

2.5. Protein Concentration. The protein concentration of the solutions was determined by the Bio-Rad Protein Assay using bovine serum albumin as a standard (Bio-Rad, CA, USA). The Bio-Rad Protein Assay is a dye-binding assay in which a differential color change of a dye occurs in response to various concentrations of protein. 
TABle 1: Primers used for construction of plasmids.

\begin{tabular}{|c|c|}
\hline Oligonucleotide & Primer \\
\hline 1 KpSSB-NdeI-N & GGGCATATGGCCAGCAGAGGCGTAAAC \\
\hline 2 KpSSB-XhoI-C & GGGCTCGAGTTAGAACGGGATGTCGTC \\
\hline 3 StSSB-NdeI-N & CTGAACATATGGCCAGCAGAGGCGTAA \\
\hline 4 StSSB-XhoI-C & TGGAACTCGAGTTAGAACGGAATGTCG \\
\hline 5 PaSSB-NdeI-N & TTGCTCATATGGCCCGTGGGGTTAACA \\
\hline 6 PaSSB-XhoI-C & TTGCACTCGAGTTAGAACGGAATGTCG \\
\hline 7 KpSSB(D91E/Q92L-SacI)-N & AAGTGGACCGAGCTCTCCGGTCAGGACA \\
\hline 8 KpSSB(D91E/Q92L-SacI)-C & GTCCTGACCGGAGAGCLTCGGTCCACTT \\
\hline 9 StSSB(D91E/Q92L-SacI)-N & AAGTGGACCGAGCTCAGTGGCCAGGAA \\
\hline 10 StSSB(D91E/Q92L-SacI)-C & TTCCTGGCCACTGAGCTCGGTCCACTT \\
\hline 11 PaSSB(G90E/Q91L-SacI)-N & AAGTGGCAGGAGCTCGACGGTCAGGAT \\
\hline $12 \mathrm{PaSSB}(\mathrm{G} 90 \mathrm{E} / \mathrm{Q} 91 \mathrm{~L}-\mathrm{SacI})-\mathrm{C}$ & ATCCTGACCGTCGAGCTCCTGCCACTT \\
\hline $13 \mathrm{KpSSBnStSSBc(E91D/L92Q)-N}$ & AAGTGGACCGATCAGAGTGGCCAGGAA \\
\hline $14 \mathrm{KpSSBnStSSBc(E91D/L92Q)-C}$ & TTCCTGGCCACTCTGATCGGTCCACTT \\
\hline $15 \mathrm{KpSSBnPaSSBc(E91D/L92Q)-N}$ & AAGTGGACCGATCAGGACGGTCAGGAT \\
\hline $16 \mathrm{KpSSBnPaSSBc(E91D/L92Q)-C}$ & ATCCTGACCGTCCTGATCGGTCCACTT \\
\hline
\end{tabular}

A fragment containing the coding sequence of KpSSB, StSSB, and PaSSB (with the stop codon) was cloned into the pET21b vector (using Primers 1-6). During the process, NdeI and XhoI restriction sites were introduced at the $5^{\prime}$-end and the $3^{\prime}$-end of these genes, after which they were ligated into the pET21b vector. To obtain the vectors for expression of the chimeric proteins KpSSBnStSSBc and KpSSBnPaSSBc, pET21b-KpSSB (Primers 7 and 8), pET21b-StSSB (Primers 9 and 10), and pET21b-PaSSB (Primers 11 and 12) vectors were mutated to create a desired SacI site. The D91E/Q92L-engineered pET21b-KpSSB vector, the D91E/Q92L-engineered pET21b-StSSB vector, and the G90E/Q91L-engineered pET21b-PaSSB vector were cut at NdeI and SacI sites. Subsequently, the KpSSBn, StSSBc-pET21b, and PaSSBc-pET21b fragments were purified. KpSSBn was ligated with StSSBc-pET21b and PaSSBc-pET21b fragments to generate the engineered pET21b-KpSSBnStSSBc and pET21b-KpSSBnPaSSBc vectors. To avoid artificial residues, positions 91 and 92 of the two plasmids were mutated back (Primers 13 to 16) to obtain pET21b-KpSSBnStSSBc and pET21b-KpSSBnPaSSBc vectors. Thus, pET21b-KpSSBnStSSBc and pET21b-KpSSBnPaSSBc will express KpSSB1-91 fused StSSB92-176 and PaSSB91-165, respectively. These plasmids were verified by DNA sequencing. Underlined nucleotides indicate the designated site for mutation or the restriction site.

2.6. Gel-Filtration Chromatography. Gel-filtration chromatography was carried out by the AKTA-FPLC system (GE Healthcare Bio-Sciences, Piscataway, NJ, USA). Briefly, purified protein $(2 \mathrm{mg} / \mathrm{mL})$ was applied to a Superdex $200 \mathrm{HR}$ 10/30 column (GE Healthcare Bio-Sciences, Piscataway, NJ, USA) equilibrated with Buffer D. The column was operated at a flow rate of $0.5 \mathrm{~mL} / \mathrm{min}$, and $0.5 \mathrm{~mL}$ fractions were collected. The proteins were detected by measuring the absorbance at $280 \mathrm{~nm}$. The column was calibrated with proteins of known molecular weight: thyroglobulin $(670 \mathrm{kDa}), \gamma$ globulin $(158 \mathrm{kDa})$, ovalbumin $(44 \mathrm{kDa})$, and myoglobin $(17 \mathrm{kDa})$. The $K_{\mathrm{av}}$ values for the standard proteins and the SSB variants were calculated from the equation: $K_{\mathrm{av}}=\left(V_{e}-\right.$ $\left.V_{o}\right) /\left(V_{c}-V_{o}\right)$, where $V_{o}$ is column void volume, $V_{e}$ is elution volume, and $V_{c}$ is geometric column volume.

2.7. Electrophoretic Mobility Shift Assay (EMSA). EMSA [52] for these SSBs was carried out by the protocol described previously for DnaB [63], PriB [59, 64-66], DnaT [57, 67], and SSB proteins $[40,42,43,52]$. Briefly, radiolabeling of various lengths of ssDNA oligonucleotides was carried out with $\left[\gamma^{32} \mathrm{P}\right]$ ATP $(6000 \mathrm{Ci} / \mathrm{mmol}$; PerkinElmer Life Sciences, Waltham, MA) and T4 polynucleotide kinase (Promega, Madison, WI, USA). The protein (0, 19, 37, 77, 155, 310, 630, 1250,2500 , and $5000 \mathrm{nM}$ ) was incubated for $30 \mathrm{~min}$ at $25^{\circ} \mathrm{C}$ with $1.7 \mathrm{nM}$ DNA substrates (dT15-65) in a total volume of $10 \mu \mathrm{L}$ in $20 \mathrm{mM}$ Tris- $\mathrm{HCl} \mathrm{pH} 8.0$ and $100 \mathrm{mM} \mathrm{NaCl}$. Aliquots $(5 \mu \mathrm{L})$ were removed from each of the reaction solutions and added to $2 \mu \mathrm{L}$ of gel-loading solution ( $0.25 \%$ bromophenol blue and $40 \%$ sucrose). The resulting samples were resolved on a native $8 \%$ polyacrylamide gel at $4^{\circ} \mathrm{C}$ in TBE buffer ( $89 \mathrm{mM}$ Tris borate and $1 \mathrm{mM}$ EDTA) for $1 \mathrm{~h}$ at $100 \mathrm{~V}$ and were visualized by autoradiography. Complexed and free DNA bands were scanned and quantified.

2.8. DNA-Binding Ability. The ssDNA-binding ability ([Protein $]_{50} ; K_{d \text {,app }}$ ) for the protein was estimated from the protein concentration that binds $50 \%$ of the input ssDNA [52]. Each [Protein $]_{50}$ is calculated as the average of three measurements $\pm \mathrm{SD}$.

2.9. Bioinformatics. Sequence alignment of KpSSB, StSSB, and PaSSB was generated by CLUSTALW2 [68]. The structure of the C-terminal domain of these SSBs was modeled by $(\mathrm{PS})^{2}$ (http://140.113.239.111/ ps2v2/docs.php/). The structures were visualized by using the program PyMol.

\section{Results}

3.1. Sequence Analysis. Based on the nucleotide sequence found, using a database search through the National Center for Biotechnology Information (NCBI), we predicted that $\mathrm{KpSSB}, \mathrm{StSSB}$, and PaSSB monomer proteins have lengths of 174, 176, and 165 amino acid residues, respectively. The size of the ssDNA-binding site of His-tagged KpSSB [40], StSSB 


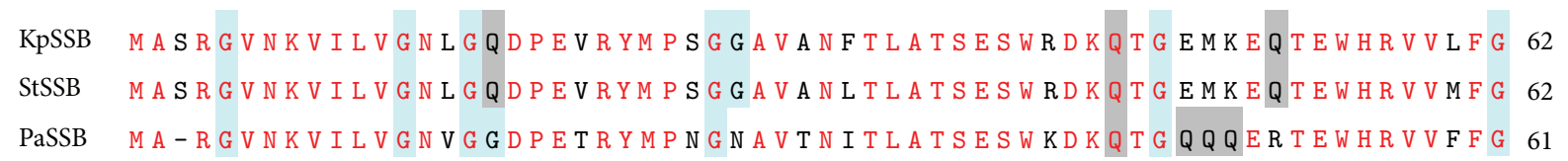

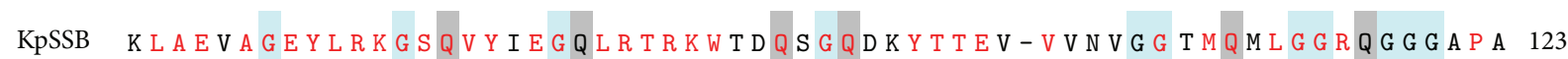
StSSB KLAEVAGEYLRGSQVYIEGQLRTRKWDQSGQERYTTEINVPQIGGVMQMLGGRGGGAPA 124 PaSsB RLAEIAGEYLRKG QVYVEGSLRTRWQGQDGQDRYTTEI-VVDINGNMQL G GR-- - - P S 120

KpSSB GGGQQQGGWGQPQQPQ- - - GGNQFSGGAQSRPQQQAPAAPSNEPPMD - FDDDIPF

StSSB G-GQQQGGWGQPQQPQQPQGGNQFSGGAQSRPQQSAP-APSNEPPMD-FDDDIPF 176

PaSSB G-DDSQRAPREPMQRP---- - Q QAPQQQSRAPQQQPAPQPAQDYD SFDDIPF 165

FIGURE 2: Multiple amino acid sequence alignment of SSB proteins. Sequence alignment of KpSSB, StSSB, and PaSSB was generated by CLUSTALW2. Identical amino acid residues are colored in red. Gly and Gln residues are shaded in cyan and gray. The N-terminal domains of these SSBs are significantly conserved.

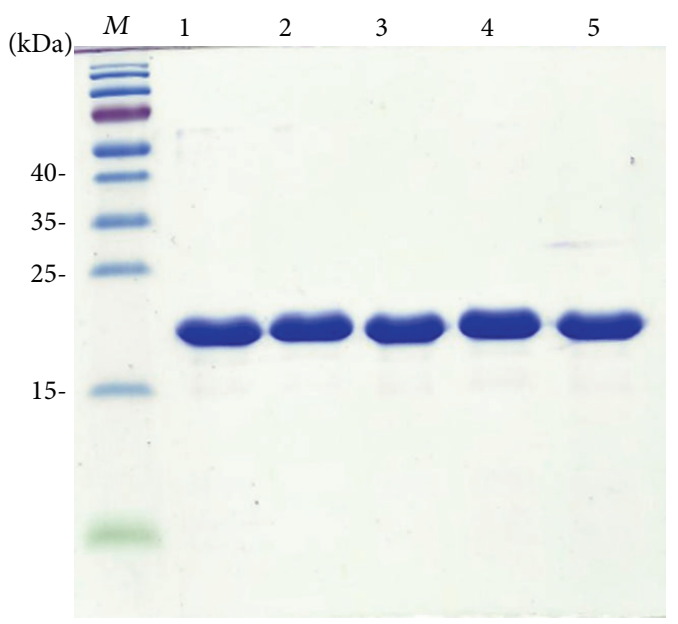

FIgURE 3: Protein purity. Coomassie Blue-stained SDS-PAGE (15\%) of the purified KpSSB (lane 1), StSSB (lane 2), PaSSB (lane 3), KpSSBnStSSBc (lane 4), KpSSBnPaSSBc (lane 5), and molecular mass standards $(M)$ are shown. The sizes of the standard proteins, from the top down, are as follows: 55, 40, 35, 25, 15, and $10 \mathrm{kDa}$. The purified SSBs migrated between the 25 and $15 \mathrm{kDa}$ standards on the SDS-PAGE.

[43], and PaSSB [42] was determined to be $26 \pm 1,22 \pm 1$, and $29 \pm 1 \mathrm{nt}$, respectively. The longer the length of the polypeptide chain, the smaller the size for ssDNA binding. Analysis of the primary structures of KpSSB, StSSB, and PaSSB by RPSBLAST revealed the presence of a putative OB-fold domain that is common to all known SSBs. Figure 2 shows that the alignments of the amino acid sequences of KpSSB, StSSB, and PaSSB amino acid residues in their N-terminal domains are highly conserved (colored in red). In the E. coli SSB-ssDNA complex [11], four essential aromatic residues, namely, Trp40, Trp54, Phe60, and Trp88, participate in ssDNA binding via stacking interactions [11]. These residues are conserved in most SSB families, including KpSSB, StSSB, and PaSSB. The important motif in the C-terminal tail of E. coli SSB, DDDIPF residues, is also conserved in KpSSB, StSSB, and PaSSB. By contrast to those motifs, the residues found in the glycinerich hinge of E. coli SSB are not conserved in KpSSB, StSSB, and PaSSB (Figure 2). Thus, the length and composition of the amino acid residues in the glycine-rich hinge may be responsible for the different ssDNA-binding site sizes of SSBs.

3.2. Expression and Purification of KpSSB, StSSB, and PaSSB. The N-terminal ssDNA-binding domain of SSB has been well-established to be highly conserved. However, SSBs possessing different ssDNA-binding site sizes have been reported. The reason that SSBs have similar ssDNA-binding domains but possess varying ssDNA-binding site sizes remains unclear. Although the ssDNA-binding site sizes of $\mathrm{KpSSB}$, StSSB, and PaSSB have been reported, we reinvestigated the ssDNA-binding properties of KpSSB, StSSB, and PaSSB in the absence of a His tag to avoid the unknown effect of a His tag (hexahistidine) on the ssDNA binding of SSB.

3.3. KpSSB Bound to ssDNA. To investigate the length of nucleotides sufficient for the formation of the KpSSB-ssDNA complex and the ssDNA-binding ability of KpSSB, we studied the binding of KpSSB to dT20 (Figure 4(a)), dT25 (Figure 4(b)), dT35 (Figure 4(c)), dT45 (Figure 4(d)), dT50 (Figure 4(e)), dT55 (Figure 4(f)), and dT60 (Figure 4(g)) with different protein concentrations. As shown in Figure 4(a), no band shift was observed when KpSSB was incubated with dT20, indicating that KpSSB could not form a stable complex with this homopolymer. By contrast to dT20, longer dT homopolymers, which include dT25-50, produced a significant band shift (C, complex), that is, formation of a stable protein-DNA complex in solution. Furthermore, two different complexes for dT55 were formed by KpSSB (Figure 4(f)). At lower protein concentrations, KpSSB formed a single complex (C1) with dT55, similar to that observed with dT50 (Figure 4(e)). However, when the KpSSB concentration was increased, another slower migrating complex (C2) was observed. 


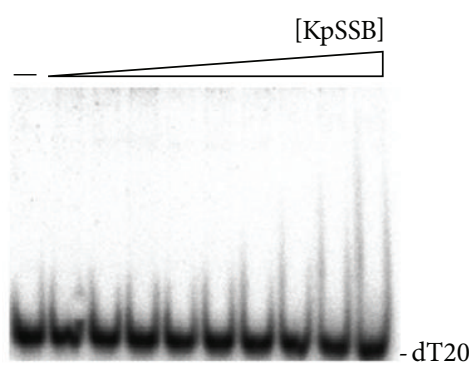

(a)

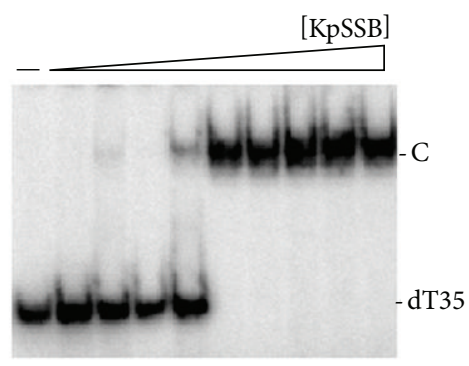

(c)

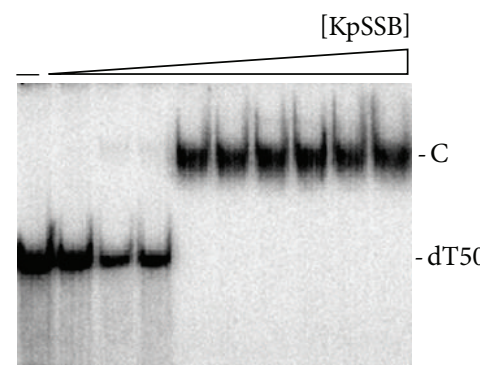

(e)

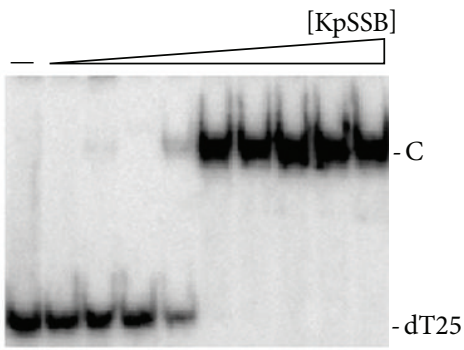

(b)

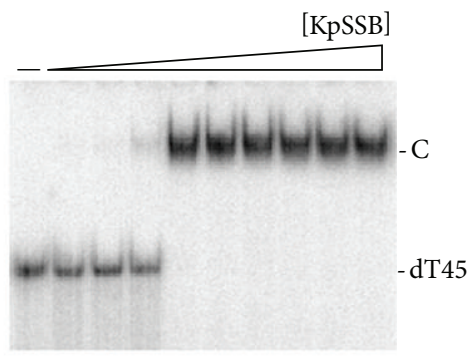

(d)

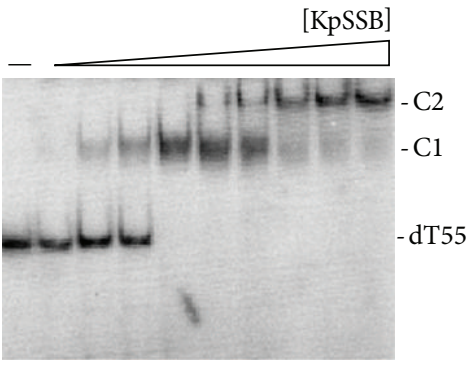

(f)

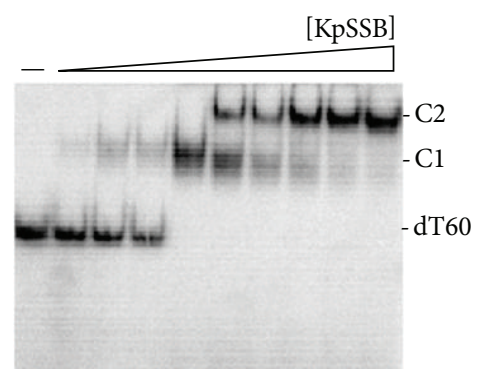

(g)

Figure 4: Binding of $\operatorname{KpSSB}$ to dT20-60. $\operatorname{KpSSB}(0,19,37,77,155,310,630,1250,2500$, and $5000 \mathrm{nM})$ was incubated for $30 \mathrm{~min}$ at $25^{\circ} \mathrm{C}$ with $1.7 \mathrm{nM}$ of (a) dT20, (b) dT25, (c) dT35, (d) dT45, (e) dT50, (f) dT55, or (g) dT60 in a total volume of $10 \mu \mathrm{L}$ in $20 \mathrm{mM}$ Tris- HCl pH 8.0 and $100 \mathrm{mM} \mathrm{NaCl}$. Aliquots $(5 \mu \mathrm{L})$ were removed from each reaction solution and added to $2 \mu \mathrm{L}$ of gel-loading solution $(0.25 \%$ bromophenol blue and $40 \%$ sucrose). The resulting samples were resolved on a native $8 \%$ polyacrylamide gel at $4^{\circ} \mathrm{C}$ in TBE buffer $(89 \mathrm{mM}$ Tris borate and $1 \mathrm{mM}$ EDTA) for $1 \mathrm{~h}$ at $100 \mathrm{~V}$ and visualized by autoradiography. Complexed and free DNA bands were scanned and quantified.

Two different complexes of KpSSB were also observed to bind to dT60 (Figure $4(\mathrm{~g})$ ). The appearance of the second complex resulted from the increased KpSSB concentration, suggesting that two KpSSB proteins may be present per oligonucleotide. Although dT55 is only $5 \mathrm{nt}$ longer than dT50 is, the presence of an extra $5 \mathrm{nt}$ in dT55 compared with that of $\mathrm{dT} 50$ provides enough interaction space for the binding of two KpSSB proteins. Therefore, one KpSSB occupies $25(50 / 2=25)$ nt to $27.5(55 / 2=27.5)$ nt of the ssDNA. The EMSA results suggest that the length of an ssDNA (or the binding site size) [52] required for KpSSB binding is $26 \pm 2 \mathrm{nt}$.

3.4. StSSB Bound to ssDNA. The binding of StSSB to dT15 (Figure 5(a)), dT20 (Figure 5(b)), dT30 (Figure 5(c)), dT40 (Figure 5(d)), dT45 (Figure 5(e)), and dT50 (Figure 5(f)) was 


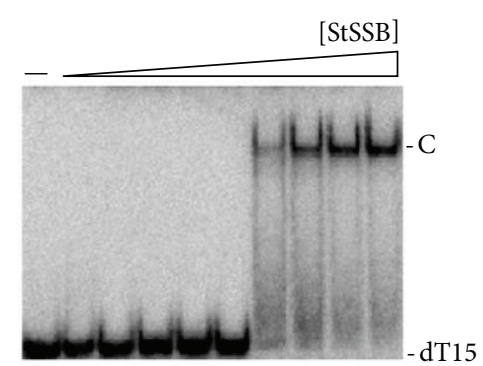

(a)

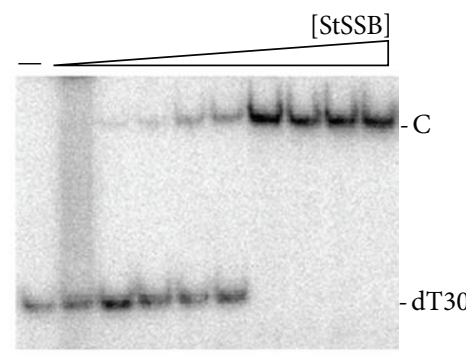

(c)

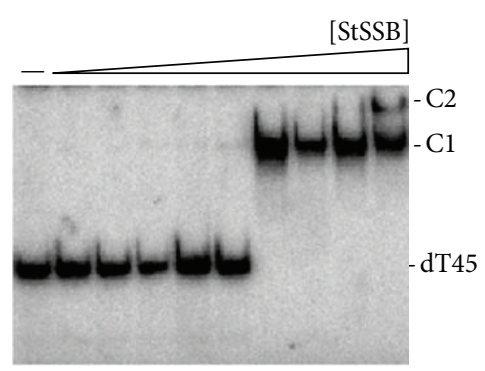

(e)

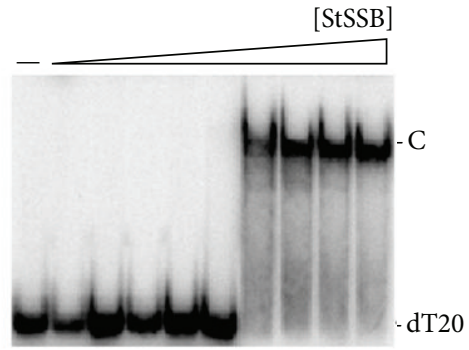

(b)

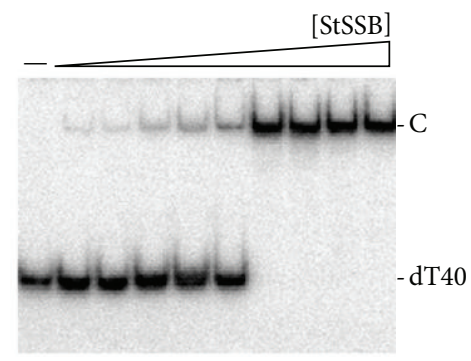

(d)

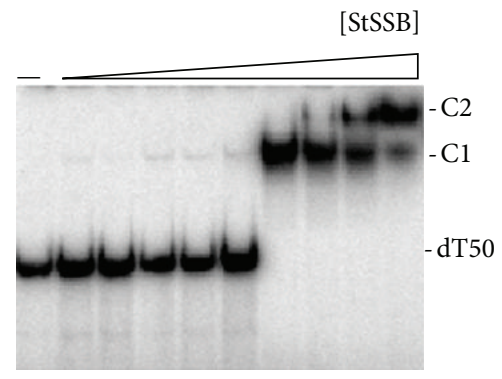

(f)

Figure 5: Binding of StSSB to dT15-50. StSSB $(0,19,37,77,155,310,630,1250,2500$, and $5000 \mathrm{nM})$ was incubated for 30 min at $25^{\circ} \mathrm{C}$ with $1.7 \mathrm{nM}$ of (a) dT15, (b) dT20, (c) dT30, (d) dT40, (e) dT45, or (f) dT50 in a total volume of $10 \mu \mathrm{L}$ in $20 \mathrm{mM}$ Tris- $\mathrm{HCl} \mathrm{pH} 8.0$ and $100 \mathrm{mM}$ $\mathrm{NaCl}$. Aliquots $(5 \mu \mathrm{L})$ were removed from each reaction solution and added to $2 \mu \mathrm{L}$ of gel-loading solution $(0.25 \%$ bromophenol blue and $40 \%$ sucrose). The resulting samples were resolved on a native $8 \%$ polyacrylamide gel at $4{ }^{\circ} \mathrm{C}$ in TBE buffer $(89 \mathrm{mM}$ Tris borate and $1 \mathrm{mM}$ EDTA) for $1 \mathrm{~h}$ at $100 \mathrm{~V}$ and visualized by autoradiography. Complexed and free DNA bands were scanned and quantified.

examined using EMSA. StSSB can bind and form a single complex with dT15 (Figure 5(a)) and dT20 (Figure 5(b)), but KpSSB cannot (Figure 4(a)). StSSB bound to dT15-40 and formed a single complex. For dT45 and dT50, two different complexes of StSSB appeared at high protein concentrations (Figures 5(e) and 5(f)). Therefore, one StSSB occupies 20 $(40 / 2=20)$ nt to $22.5(45 / 2=22.5)$ nt of the ssDNA. The EMSA results suggest that the length of an ssDNA (or the binding site size) [52] required for StSSB binding is $21 \pm 2 \mathrm{nt}$.

3.5. PaSSB Bound to ssDNA. The binding of PaSSB to dT20 (Figure 6(a)), dT25 (Figure 6(b)), dT35 (Figure 6(c)), dT45 (Figure 6(d)), dT55 (Figure 6(e)), dT60 (Figure 6(f)), and dT65 (Figure 6(g)) was studied by EMSA. Unlike StSSB, no complex was observed when PaSSB was incubated with dT20. Some smears were observed, indicating that PaSSB interacts with dT20. However, the ssDNA may be too short to be fully wrapped by PaSSB. PaSSB could form a single complex with dT25-55 and form two distinct complexes with dT60 and dT65 (Figures 6(f) and 6(g)), respectively. Therefore, one PaSSB occupies $27.5(55 / 2=27.5)$ nt to $30(60 / 2=30) \mathrm{nt}$ of the ssDNA. These results from EMSA suggest that the length of an ssDNA (or the binding site size) [52] required for PaSSB binding is $29 \pm 2 \mathrm{nt}$. Although the SSBs, that is, KpSSB, StSSB, and PaSSB, have significantly similar ssDNA-binding domains, their binding site sizes are different and range from 19 (21 \pm 2 ; StSSB $)$ to $31(29 \pm 2$; PaSSB $)$ nt. The obtained EMSA results (Figures 4-6) also show that the binding site sizes of the untagged SSBs (KpSSB, StSSB, and PaSSB) were found to be almost identical to those of the His-tagged ones $[40,42,43]$.

3.6. Design of the Chimeric KpSSB Proteins KpSSBnStSSBc and $K p S S B n P a S S B c$. The N-terminal ssDNA-binding domain of KpSSB, StSSB, and PaSSB is highly conserved (Figure 2), but their binding site sizes are different (Figures 4-6) and range from $19 \mathrm{nt}$ to $31 \mathrm{nt}$. The C-terminal acidic tails, DDDIPF, are conserved (Figure 2), and these features led us to assess 


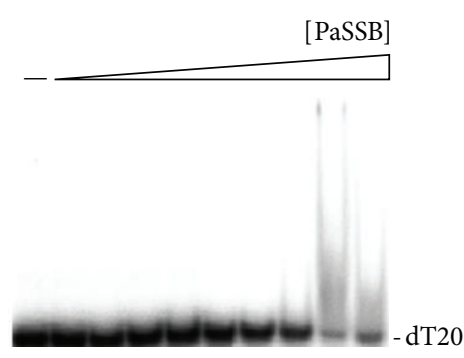

(a)

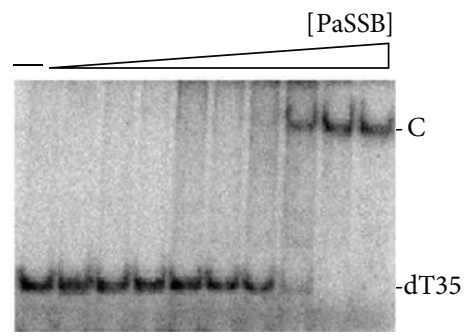

(c)

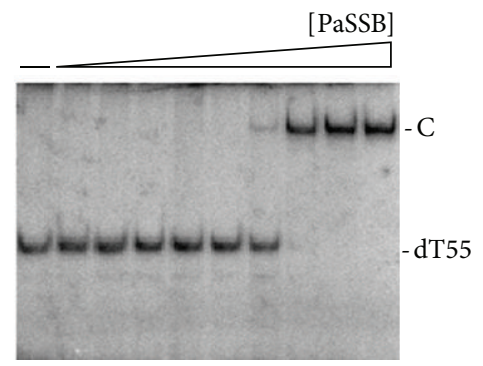

(e)

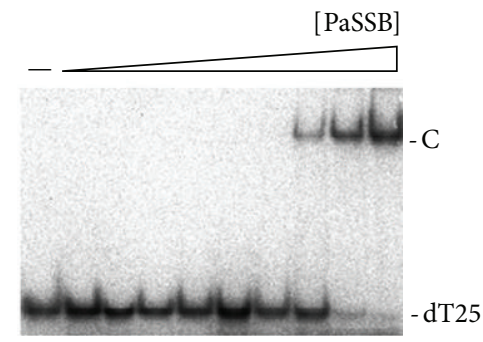

(b)

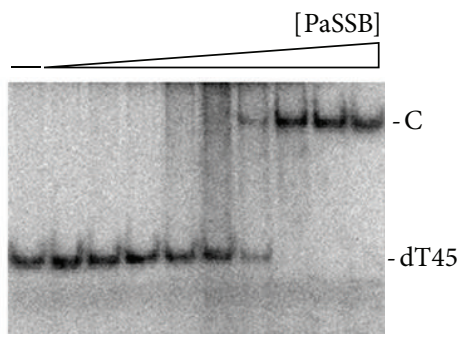

(d)

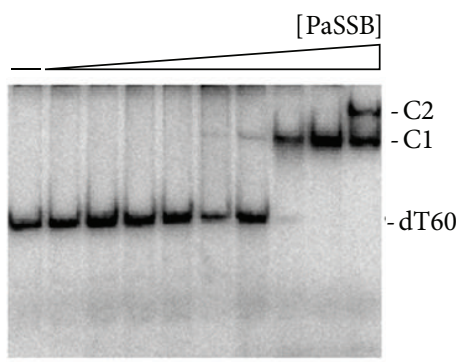

(f)

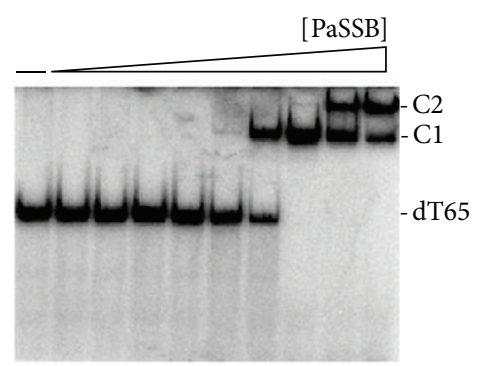

(g)

Figure 6: Binding of PaSSB to dT20-65. PaSSB $(0,19,37,77,155,310,630,1250,2500$, and $5000 \mathrm{nM})$ was incubated for 30 min at $25^{\circ} \mathrm{C}$ with $1.7 \mathrm{nM}$ of (a) dT20, (b) dT25, (c) dT35, (d) dT45, (e) dT55, (f) dT60, or (g) dT65 in a total volume of $10 \mu \mathrm{L}$ in $20 \mathrm{mM}$ Tris- $\mathrm{HCl}$ pH 8.0 and $100 \mathrm{mM} \mathrm{NaCl}$. Aliquots $(5 \mu \mathrm{L})$ were removed from each reaction solution and added to $2 \mu \mathrm{L}$ of gel-loading solution $(0.25 \%$ bromophenol blue and $40 \%$ sucrose). The resulting samples were resolved on a native $8 \%$ polyacrylamide gel at $4^{\circ} \mathrm{C}$ in TBE buffer $(89 \mathrm{mM}$ Tris borate and $1 \mathrm{mM}$ EDTA) for $1 \mathrm{~h}$ at $100 \mathrm{~V}$ and visualized by autoradiography. Complexed and free DNA bands were scanned and quantified.

whether the flexible glycine-rich hinge in the C-terminal domain, which is not conserved among SSBs, is involved in the determination of the binding site size of SSB. Thus, the C-terminal domains of StSSB and PaSSB were swapped with KpSSB through protein chimeragenesis.

3.7. KpSSBnStSSBc Bound to ssDNA. The binding of KpSSBnStSSBc to dT15 (Figure 7(a)), dT20 (Figure 7(b)), dT40 (Figure 7(c)), and dT45 (Figure 7(d)) was examined using EMSA. KpSSBnStSSBc exhibited significantly different
ssDNA-binding properties from those of KpSSB. Unlike KpSSB (Figure 4), both KpSSBnStSSBc (Figure 8) and StSSB (Figure 5) can bind and form a single complex with dT15 and dT20. Similar to StSSB, KpSSBnStSSBc binds to dT15-40 and forms a single complex. For dT45, two different complexes of KpSSBnStSSBc appeared at high protein concentrations (Figure $8(\mathrm{~d})$ ); this EMSA feature was also similar to that of StSSB. One KpSSBnStSSBc occupies $20(40 / 2=20)$ nt to $22.5(45 / 2=22.5) n t$ of the ssDNA. These EMSA results suggest that the length of an ssDNA (or the binding site 


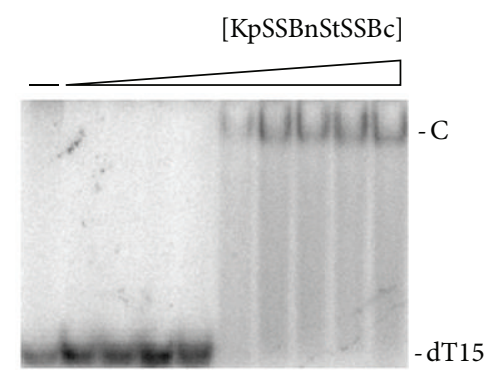

(a)

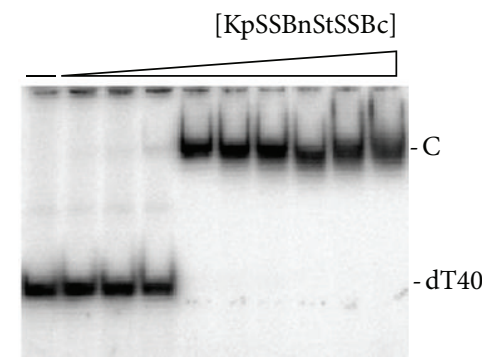

(c)

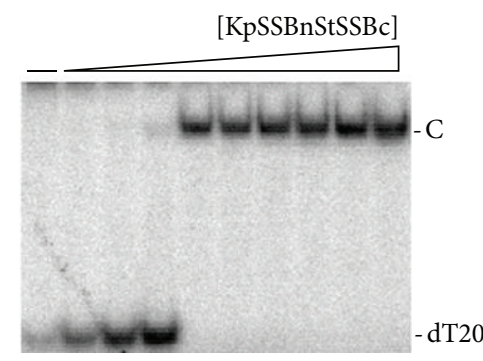

(b)

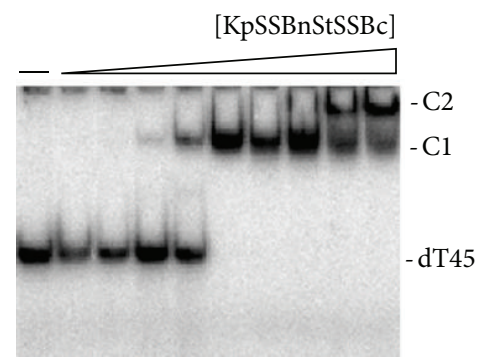

(d)

Figure 7: Binding of KpSSBnStSSBc to dT15-45. KpSSBnStSSBc (0, 19, 37, 77, 155, 310, 630, 1250, 2500, and $5000 \mathrm{nM})$ was incubated for $30 \mathrm{~min}$ at $25^{\circ} \mathrm{C}$ with $1.7 \mathrm{nM}$ of (a) dT15, (b) dT20, (c) dT40, or (d) dT45 in a total volume of $10 \mu \mathrm{L}$ in $20 \mathrm{mM}$ Tris- $\mathrm{HCl} \mathrm{pH} 8.0$ and $100 \mathrm{mM}$ $\mathrm{NaCl}$. Aliquots $(5 \mu \mathrm{L})$ were removed from each reaction solution and added to $2 \mu \mathrm{L}$ of gel-loading solution $(0.25 \%$ bromophenol blue and $40 \%$ sucrose). The resulting samples were resolved on a native $8 \%$ polyacrylamide gel at $4^{\circ} \mathrm{C}$ in TBE buffer $(89 \mathrm{mM}$ Tris borate and $1 \mathrm{mM}$ EDTA) for $1 \mathrm{~h}$ at $100 \mathrm{~V}$ and visualized by autoradiography. Complexed and free DNA bands were scanned and quantified.

size) [52] required for KpSSBnStSSBc binding is $21 \pm 2 \mathrm{nt}$, a value identical to that for StSSB (Figure 5). Swapping of the C-terminal domain of StSSB with KpSSB changes the size of the ssDNA-binding site from $26 \mathrm{nt}$ to $21 \mathrm{nt}$.

3.8. KpSSBnPaSSBc Bound to ssDNA. The binding features of KpSSBnPaSSBc with dT20 (Figure 8(a)), dT25 (Figure 8(b)), dT40 (Figure 8(c)), dT55 (Figure 8(d)), and dT60 (Figure $8(\mathrm{e})$ ) were studied by EMSA. Similar to the cases of KpSSB and PaSSB, no complex was observed when KpSSBnPaSSBc was incubated with dT20. However, KpSSB$\mathrm{nPaSSB}$ c still exhibited dramatically different ssDNA-binding properties from those of KpSSB. KpSSB can form two distinct complexes with dT55 (Figure 4(f)), but both KpSSBnPaSSBc (Figure 9) and PaSSB (Figure 6) cannot. One KpSSBnPaSSBc occupies $27.5(55 / 2=27.5)$ nt to $30(60 / 2=30) \mathrm{nt}$ of the ssDNA. The above EMSA results suggest that the length of an ssDNA (or the binding site size) [52] required for $\mathrm{KpSSBnPaSSBc}$ binding is $29 \pm 2 \mathrm{nt}$, a value identical to that of PaSSB. Swapping of the C-terminal domain of PaSSB to KpSSB changes the size of the ssDNA-binding site from $26 \mathrm{nt}$ to $29 \mathrm{nt}$. Although these SSBs, namely, KpSSB, StSSB, PaSSB, $\mathrm{KpSSBnStSSBc}$, and $\mathrm{KpSSBnPaSSBc}$, have nearly identical ssDNA-binding domains, their binding site sizes are different (Table 2). Thus, the size of the ssDNA-binding site required for second SSB binding is likely to be dependent on the Cterminal domain of SSB.
3.9. Binding Constants of the SSB-ssDNA Complexes Determined from EMSA. To compare the ssDNA-binding abilities of KpSSB, StSSB, PaSSB, KpSSBnStSSBc, and KpSS$\mathrm{BnPaSSBc}$, the midpoint values for input ssDNA binding, calculated from the titration curves of EMSA and referred to as [Protein $]_{50}$ (monomer), were quantified and are summarized in Table 2. Although the N-terminal ssDNAbinding domains of these SSB proteins are highly similar (Figure 2), their ssDNA-binding activities and binding site sizes are different (Table 2 ). $[\mathrm{KpSSB}]_{50}$ values ranged from $100 \mathrm{nM}$ to $220 \mathrm{nM}$; [StSSB] $]_{50}$ values ranged from $420 \mathrm{nM}$ to $650 \mathrm{nM}$; $[\mathrm{PaSSB}]_{50}$ values ranged from $550 \mathrm{nM}$ to $1700 \mathrm{nM}$; $[\mathrm{KpSSBnStSSBc}]_{50}$ values ranged from $110 \mathrm{nM}$ to $260 \mathrm{nM}$; and KpSSBnPaSSBc$_{50}$ values ranged from $220 \mathrm{nM}$ to $390 \mathrm{nM}$. The ssDNA-binding ability is as follows, in the order of decreasing affinity: KpSSB > KpSSBnStSSBc > KpSSBn$\mathrm{PaSSBC}>\mathrm{StSSB}>\mathrm{PaSSB}$. Results from the above analyses indicate that the exchange of the C-terminal domain in SSB significantly changed the ssDNA-binding ability and the DNA-binding behavior (complex number). The reason as to why swapping of the C-terminal domain can affect the ssDNA-binding activity of SSB remains unclear. The C-terminal domain of SSB is suggested to be involved in ssDNA binding. However, this relation is not evident in the results of the cocrystal structure.

3.10. Oligomeric State of KpSSBnStSSBc and KpSSBnPaSSBc in Solution. Gel-filtration chromatography was used to confirm 


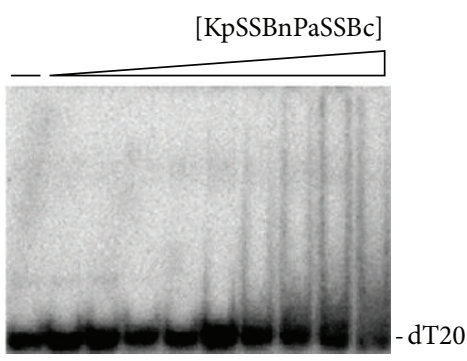

(a)

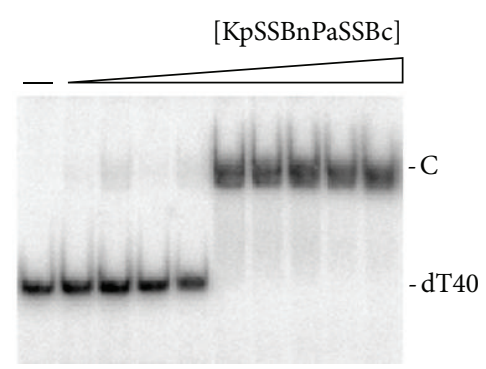

(c)

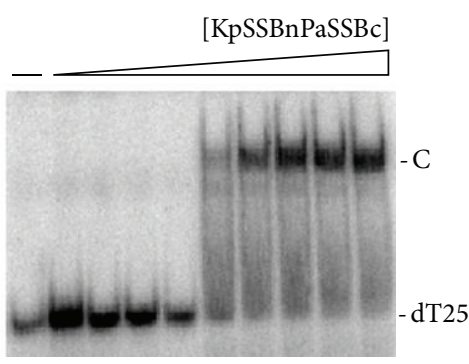

(b)

$[\mathrm{KpSSBnPaSSBc}]$

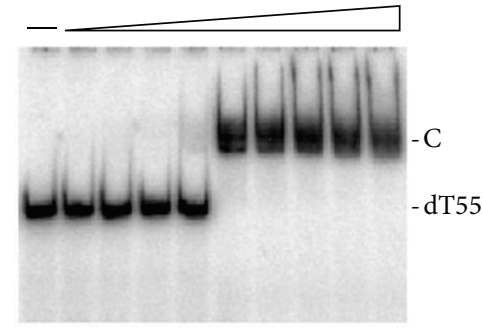

(d)

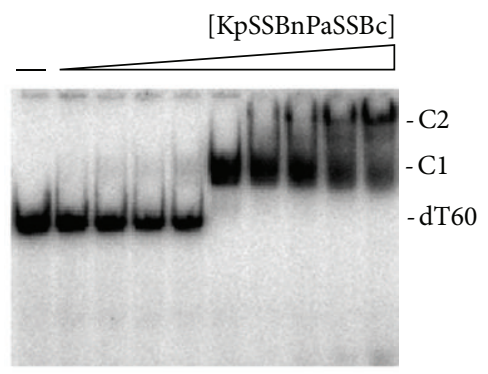

(e)

Figure 8: Binding of KpSSBnPaSSBc to dT20-60. KpSSBnPaSSBc (0, 19, 37, 77, 155, 310, 630, 1250, 2500, and $5000 \mathrm{nM})$ was incubated for $30 \mathrm{~min}$ at $25^{\circ} \mathrm{C}$ with $1.7 \mathrm{nM}$ of (a) dT20, (b) dT25, (c) dT40, (d) dT55, or (e) dT60 in a total volume of $10 \mu \mathrm{L}$ in $20 \mathrm{mM}$ Tris- $\mathrm{HCl} \mathrm{pH} 8.0$ and $100 \mathrm{mM} \mathrm{NaCl}$. Aliquots $(5 \mu \mathrm{L})$ were removed from each reaction solution and added to $2 \mu \mathrm{L}$ of gel-loading solution $(0.25 \%$ bromophenol blue and $40 \%$ sucrose). The resulting samples were resolved on a native $8 \%$ polyacrylamide gel at $4^{\circ} \mathrm{C}$ in TBE buffer $(89 \mathrm{mM}$ Tris borate and $1 \mathrm{mM}$ EDTA) for $1 \mathrm{~h}$ at $100 \mathrm{~V}$ and visualized by autoradiography. Complexed and free DNA bands were scanned and quantified.

that the oligomeric state of KpSSBnStSSBc and KpSSBn$\mathrm{PaSSB} c$ remains as tetramers after chimeragenesis. The analysis of purified KpSSBnStSSBc and KpSSBnPaSSBc ( $2 \mathrm{mg} / \mathrm{mL})$ using a Superdex $200 \mathrm{HR}$ 10/30 column revealed a single peak with elution volumes of 78.6 and $78.9 \mathrm{~mL}$, respectively. Assuming that KpSSBnStSSBc and KpSSBnPaSSBc both have shapes and partial specific volumes similar to the standard proteins, the native molecular masses of KpSSBnStSSBc and $\mathrm{KpSSBnPaSSBc}$ were estimated to be 76641 and $74827 \mathrm{Da}$, as calculated from a standard linear regression equation, $K_{\mathrm{av}}=$ $-0.3684(\log \mathrm{Mw})+2.2707$ (Figure 9). The native molecular masses for KpSSBnStSSBc and KpSSBnPaSSBc are approximately four times the mass of the monomer $(\sim 19 \mathrm{kDa})$. Therefore, KpSSBnStSSBc and KpSSBnPaSSBc under the above chromatographic conditions are stable tetramers in solution. Although the exchange of the C-terminal domain in SSB significantly changed the ssDNA-binding ability and DNA-binding behavior (complex number), protein chimeragenesis did not cause any change in the oligomeric state of SSB.
3.11. Summary of Gly, Gln, and Pro Number in SSBs. To analyze the C-terminal amino acid composition of SSBs, we further counted the number of Gly, Gln, and Pro residues in different SSB segments. SSB is abundant in Gly, Gln, and Pro (GQP) (Table 3). The GQP contents of KpSSB191, StSSB1-91, and PaSSB1-90 are similar. However, the Gly number of PaSSB116-165 is significantly lower than that of KpSSB116-174 and StSSB117-176; PaSSB116-165 contains only 1 Gly, but KpSSB116-174 and StSSB117-176 contain 11 and 12 Gly, respectively. In addition, we found different distribution patterns among KpSSB, StSSB, and PaSSB. Although they contain similar number of $G \ln (\mathrm{Q})$, the QQQ pattern is frequently found in PaSSB (Table 3).

3.12. Structural Modeling of SSBs. Given its disordered C-terminal domain, the crystal structure of the full-length SSB is lacking, even when SSB can be crystallized with DNA [69]. We attempted to model the structure by homology modeling using the bioinformatics program (PS) ${ }^{2}$ to obtain an 
TABLE 2: ssDNA binding properties of KpSSB, StSSB, PaSSB, $\mathrm{KpSSBnStSSBc}$, and $\mathrm{KpSSBnPaSSBc}$ as analyzed by EMSA.

\begin{tabular}{|c|c|c|c|}
\hline Protein & DNA & {$[\text { Protein }]_{50}(\mathrm{nM})$} & Complex number \\
\hline \multirow{7}{*}{ KpSSB } & $\mathrm{dT} 20$ & ND & 0 \\
\hline & $\mathrm{dT} 25$ & $200 \pm 20$ & 1 \\
\hline & dT35 & $220 \pm 30$ & 1 \\
\hline & $\mathrm{dT} 45$ & $100 \pm 10$ & 1 \\
\hline & dT50 & $110 \pm 20$ & 1 \\
\hline & dT55 & $100 \pm 20$ & 2 \\
\hline & dT60 & $100 \pm 10$ & 2 \\
\hline \multirow{6}{*}{ StSSB } & $\mathrm{dT} 15$ & $650 \pm 120$ & 1 \\
\hline & dT20 & $450 \pm 80$ & 1 \\
\hline & dT30 & $420 \pm 60$ & 1 \\
\hline & dT40 & $420 \pm 80$ & 1 \\
\hline & $\mathrm{dT} 45$ & $440 \pm 60$ & 2 \\
\hline & $\mathrm{dT} 50$ & $440 \pm 50$ & 2 \\
\hline \multirow{7}{*}{ PaSSB } & $\mathrm{dT} 20$ & ND & 0 \\
\hline & $\mathrm{dT} 25$ & $1700 \pm 250$ & 1 \\
\hline & dT35 & $950 \pm 180$ & 1 \\
\hline & dT45 & $780 \pm 160$ & 1 \\
\hline & dT55 & $820 \pm 90$ & 1 \\
\hline & dT60 & $810 \pm 110$ & 2 \\
\hline & dT65 & $550 \pm 70$ & 2 \\
\hline \multirow{4}{*}{ KpSSBnStSSBc } & $\mathrm{dT} 15$ & $260 \pm 60$ & 1 \\
\hline & $\mathrm{dT} 20$ & $110 \pm 20$ & 1 \\
\hline & $\mathrm{dT} 40$ & $120 \pm 20$ & 1 \\
\hline & $\mathrm{dT} 45$ & $160 \pm 20$ & 2 \\
\hline \multirow{5}{*}{$\mathrm{KpSSBnPaSSBc}$} & dT20 & ND & 0 \\
\hline & $\mathrm{dT} 25$ & $390 \pm 60$ & 1 \\
\hline & $\mathrm{dT} 40$ & $220 \pm 30$ & 1 \\
\hline & dT55 & $230 \pm 30$ & 1 \\
\hline & dT60 & $230 \pm 30$ & 2 \\
\hline
\end{tabular}

[Protein $]_{50}$ was calculated from the titration curves of EMSA by determining the concentration of the protein $(\mu \mathrm{M})$ needed to achieve the midpoint value for input ssDNA binding. For some oligonucleotides, input ssDNA binding was the sum of the intensities from the two separate ssDNA-protein complexes. Errors are standard deviations determined by three independent titration experiments.

Table 3: Summary of Gly, Gln, and Pro number in SSB.

\begin{tabular}{lccc}
\hline SSB segment & G & Q & P \\
\hline KpSSB1-91 & 10 & 5 & 2 \\
StSSB1-91 & 10 & 5 & 2 \\
PaSSB1-90 & 11 & 6 & 2 \\
\hline KpSSB92-174 & 18 & 16 & 9 \\
StSSB92-176 & 17 & 18 & 11 \\
PaSSB91-165 & 5 & 15 & 11 \\
\hline KpSSB116-174 & 11 & 12 & 9 \\
StSSB117-176 & 12 & 13 & 10 \\
PaSSB116-165 & 1 & 12 & 11 \\
\hline
\end{tabular}

in-depth understanding of the structure-function relationship of the C-terminal domains of these SSBs [70, 71].

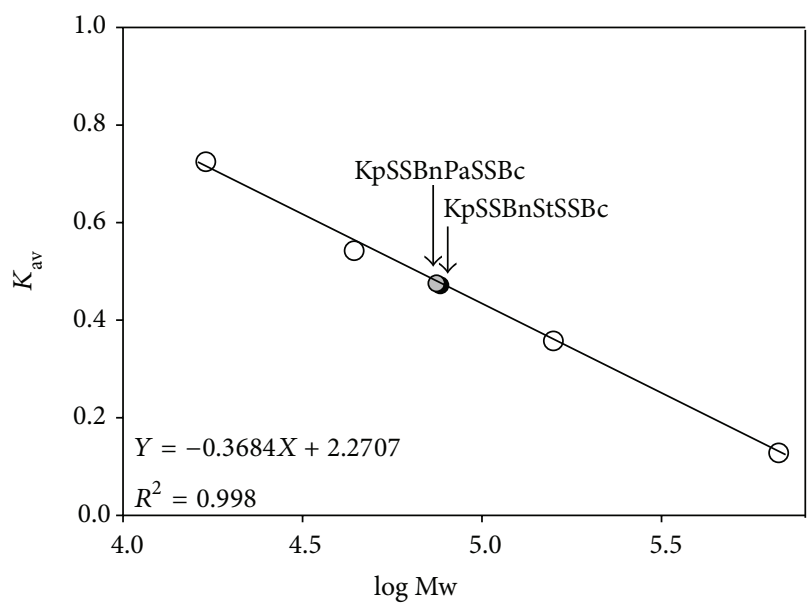

FIGURE 9: Gel-filtration chromatographic analyses of KpSSBnStSSBc and $\mathrm{KpSSBnPaSSBc}$. Purified protein $(2 \mathrm{mg} / \mathrm{mL})$ was applied to a Superdex 200 HR 10/30 column (GE Healthcare Bio-Sciences, Piscataway, NJ, USA) equilibrated with Buffer D. The column was operated at a flow rate of $0.5 \mathrm{~mL} / \mathrm{min}$, and $0.5 \mathrm{~mL}$ fractions were collected. The proteins were detected by measuring the absorbance at $280 \mathrm{~nm}$. The column was calibrated with proteins of known molecular weight: thyroglobulin $(670 \mathrm{kDa}), \gamma$-globulin $(158 \mathrm{kDa})$, ovalbumin $(44 \mathrm{kDa})$, and myoglobin $(17 \mathrm{kDa})$. The $K_{\mathrm{av}}$ values for the standard proteins and the SSB variants were calculated from the equation: $K_{\mathrm{av}}=\left(V_{e}-V_{o}\right) /\left(V_{c}-V_{o}\right)$, where $V_{o}$ is column void volume, $V_{e}$ is elution volume, and $V_{c}$ is geometric column volume.

$(\mathrm{PS})^{2}$ (http://140.113.239.111/ ps2v2/docs.php/) is an automatic homology modeling server that combines both sequence and secondary structure information to detect the homologous proteins with remote similarity and the targettemplate alignment. After pasting the amino acid sequence to the website of (PS) ${ }^{2}$, only one hit (Protein Data Bank entry: 1QVC; EcSSB) for the C-terminal domains of KpSSB and StSSB was suggested. For the C-terminal domain of PaSSB, only one hit, that is, CstF-77 (Protein Data Bank entry: 2OOE; cleavage stimulation factor, CstF), but not EcSSB, was suggested as the template for modeling. Figure 10 shows that modeled structures of these SSB C-terminal domains are highly disordered but that of PaSSB is more ordered than that of other domains.

\section{Discussion}

In this study, we examined the sizes of the binding site of the untagged SSB and the chimeric SSB from the ubiquitous opportunistic pathogens K. pneumoniae, S. enterica serovar Typhimurium LT2, and $P$. aeruginosa PAO1. Many clinical strains of the abovementioned bacteria are highly resistant to antibiotics [72-75]. The development of clinically useful small-molecule antibiotics has been a seminal event in the field of infectious diseases [48]. Nucleic acid metabolism is one of the most basic biological functions and should be a prime target in antibiotic development [76-78]. Many bacterial SSBs form conserved protein interaction "hubs" that are essential to recruit many proteins involved in DNA 


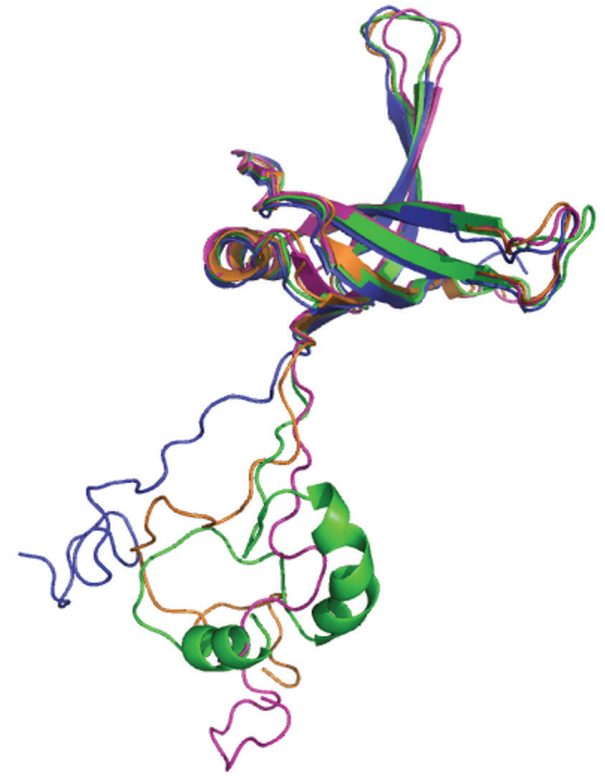

FIgURE 10: Structure modeling of SSB. The structures of KpSSB1115, StSSB1-115, and PaSSB1-115 (the N-terminal domain of SSB) were modeled by SWISS-MODEL. The structures of KpSSB116142, StSSB116-142, and PaSSB121-160 (the C-terminal domain of SSB) were modeled by (PS) ${ }^{2}$. Other regions of SSBs could not be modeled by these two programs. The structures of the $\mathrm{N}$-terminal domain and the C-terminal domain of these SSBs were manually linked (KpSSB1-142, blue; StSSB1-142, pink; PaSSB1-160, green) and superimposed with the crystal structure of EcSSB1-142 (orange) (PDB entry: 1QVC) for comparison. For clarity, only one subunit of the tetramer was shown for each SSB.

replication, recombination, and repair SSB/DNA nucleoprotein substrates [79]. Thus, SSBs may be promising targets in antibiotic development [80]. As a first step toward achieving this goal, we investigated why SSBs possess highly conserved $\mathrm{N}$-terminal ssDNA-binding domain but exhibit varying binding site sizes. One significant clue is that their flexible hinges and the length at the C-terminus are different as revealed by sequence alignment (Figure 2).

The interactions of various SSBs with ssDNA have been analyzed using a variety of techniques such as tryptophanfluorescence quenching [47], filter binding [81], EMSA [52, 82], analytical ultracentrifugation [83], electron microscopy [84], nuclease digestion [44], single-molecule fluorescence microscopy [48], and crystallographic analyses [11]. In this study, we have examined the electrophoretic mobility shift patterns of KpSSB, StSSB, PaSSB, KpSSBnStSSBc, and KpSS$\mathrm{BnPaSSBc}$ bound to different lengths of ssDNA and determined the corresponding binding site sizes to be 26,21 , 29, 22, and 29 nt per tetramer, respectively (Figures 48). PaSSB and KpSSBnPaSSBc have the largest sizes for ssDNA binding among the SSBs studied. We also identified His-tagged and untagged SSBs that have similar ssDNAbinding site sizes $[40,42,43]$. EMSA is a well-established approach in studies of molecular biology [52], and the use of radioactive tracer in this assay allows detection of the actual formation of the distinct protein-DNA complex(es) [53]. For example, DNase protection assay and footprinting assay using radioactive tracer can determine the specific DNA sequence complexed by a protein. In EMSA, when the length of the nucleotides is sufficient for the binding of two or more SSB molecules, the electrophoretic mobility of the higher SSB oligomer complex will be lower than that of the smaller SSB oligomer complex $[52,54]$. In addition, results of the ssDNAbinding site size from EMSA and cocrystal structure of SSB were consistent [27]. Thus, throughout this paper, we determined the ssDNA-binding site sizes of SSB from the EMSA behavior.

Many SSBs bind to ssDNA with some degree of positive cooperativity. Cooperativity can result from direct proteinprotein interactions between the nearest neighbors, such as the LAST motif in the T4 gene-32 protein [85] and the arginine-mediated interaction motif in Thermus SSB [86, 87]. Cooperativity can also result from the protein-induced distortion of adjacent DNA, as demonstrated in Sulfolobus $\mathrm{SSB}, \mathrm{PriB}$, and FOXK1a proteins $[23,60,88]$. In the cases of $\mathrm{KpSSB}, \mathrm{StSSB}$, and PaSSB (Figures 4-6), binding appeared to be nearly noncooperative for several DNAs because all DNA mainly shifts into the first complex $(\mathrm{C} 1)$ before the appearance of the second complex (C2) when subjected to increasing protein concentrations. The length dependence of the $[\mathrm{SSB}]_{50}$ values suggests that the amount of spacing is optimum for steric considerations (Table 2).

Because bacteria have varying genomic DNA sizes, their SSBs may need to evolve to have different binding site sizes for DNA metabolism. Results from protein chimeragenesis showed the C-terminal domain dependence of the binding site sizes of SSB (Figure 11). The experimental data showed that the binding site size of KpSSBnStSSBc was similar to that of StSSB and the size of the binding site of KpSSBnPaSSBc was similar to that of PaSSB. The reason for which the binding site size of SSB changed, followed by swapping of the C-terminal domain, remains unclear. Flexibility, number of glycine residues, and/or different QQQ patterns of the C-terminal domain of SSB (Figure 2 and Table 3 ) may be important factors for determining the ssDNA-binding site size. In fact, the C-terminal domain of PaSSB, that is, PaSSB116-165, has only 1 Gly residue, which is significantly less than that of KpSSB (11 Gly) and StSSB (12 Gly). Gly (and Pro) is an important component of the flexible region; a protein that contains low Gly content is predicted to have low flexibility. Unlike typical SSB [35, 69], PaSSB116-165 has a partial structure (Figure 10). Although KpSSB, StSSB, and PaSSB contain similar number of Gln (Q), the QQQ pattern is frequently found in PaSSB (Figure 2 and Table 3). PolyQ and repeated sequences GAGAG are commonly found in the structures of amyloids, silk fibers, and neurodegradation proteins [89-92]. Considering that the simple coil polyQ, the heptapeptide GNNQQNY, and the hexapeptide NNQQNY can cause protein aggregation and nucleation [93-95], the distribution of Gln in the C-terminal domain of a tetrameric SSB may also be an important determinant of the ssDNAbinding site size of SSB by some steric hindrances (Figure 11). However, the above speculation must be confirmed by further biochemical experiments. 

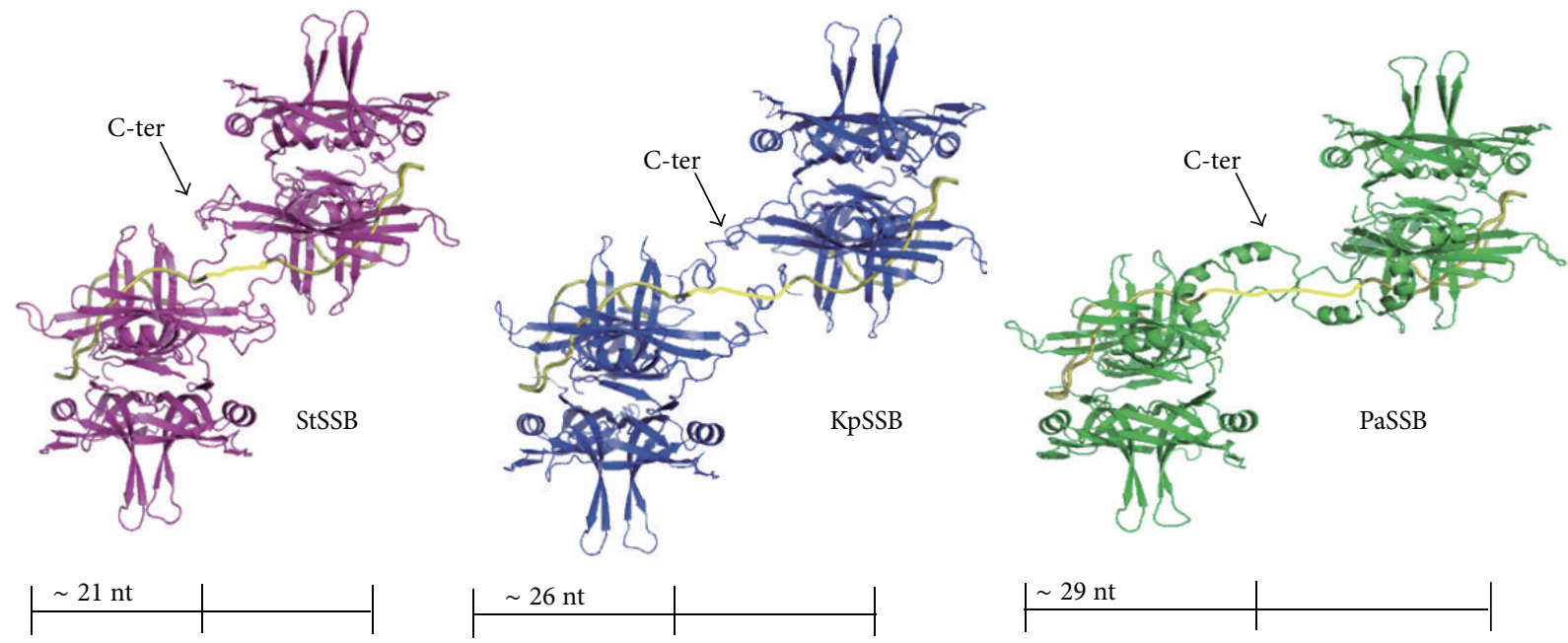

FIGURE 11: Possible models for explaining why SSBs are with different binding site sizes. Two modeled structures of KpSSB1-142 (blue), StSSB1142 (pink), and PaSSB1-160 (green) complexed with ssDNA (gold) are shown. For clarity, only one C-terminal domain was shown for each SSB tetramer. By using the electrophoretic mobility shift assay and the protein chimeragenesis, we characterized that the binding site sizes of KpSSB, StSSB, PaSSB, KpSSBnStSSBc, and KpSSBnPaSSBc were 26, 21, 29, 21, and 29 nt per tetramer, respectively. KpSSB, StSSB, and PaSSB are similar proteins whose $\mathrm{N}$-terminal ssDNA-binding domains are almost identical. Thus, the C-terminal domain of SSB may indirectly contribute to ssDNA binding and wrapping and affects the binding site size by the steric hindrance.

\section{Conclusion}

In this study, we characterized the ssDNA-binding properties of untagged SSBs from K. pneumoniae, S. enterica serovar Typhimurium LT2, and P. aeruginosa PAO1 and proposed a role of the C-terminal flexible domain for ssDNA binding from the protein chimeragenesis and EMSA results. The amino acid sequence of the N-terminal ssDNA-binding/oligomerization domain in these pathogenic SSBs is highly conserved, but their apparent binding site sizes are different. This finding indicates that the C-terminal proteinprotein interaction domain may also indirectly contribute to ssDNA binding and wrapping.

\section{Conflict of Interests}

The authors declare that there is no conflict of interests regarding the publication of this paper.

\section{Acknowledgment}

This research was supported by a Grant from the National Science Council, Taiwan (NSC 102-2320-B-040-019 to ChengYang Huang).

\section{References}

[1] R. Reyes-Lamothe, D. J. Sherratt, and M. C. Leake, "Stoichiometry and architecture of active DNA replication machinery in escherichia coli," Science, vol. 328, no. 5977, pp. 498-501, 2010.

[2] D. J. Richard, E. Bolderson, and K. K. Khanna, "Multiple human single-stranded DNA binding proteins function in genome maintenance: structural, biochemical and functional analysis,"
Critical Reviews in Biochemistry and Molecular Biology, vol. 44, no. 2-3, pp. 98-116, 2009.

[3] R. D. Shereda, A. G. Kozlov, T. M. Lohman, M. M. Cox, and J. L. Keck, "SSB as an organizer/mobilizer of genome maintenance complexes," Critical Reviews in Biochemistry and Molecular Biology, vol. 43, no. 5, pp. 289-318, 2008.

[4] D. J. Richard, E. Bolderson, L. Cubeddu et al., "Single-stranded DNA-binding protein hSSB1 is critical for genomic stability," Nature, vol. 453, no. 7195, pp. 677-681, 2008.

[5] R. R. Meyer and P. S. Laine, "The single-stranded DNA-binding protein of Escherichia coli," Microbiological Reviews, vol. 54, no. 4, pp. 342-380, 1990.

[6] C. Yang, U. Curth, C. Urbanke, and C. Kang, "Crystal structure of human mitochondrial single-stranded DNA binding protein at $2.4 \AA$ Å resolution," Nature Structural Biology, vol. 4, no. 2, pp. 153-157, 1997.

[7] G. Webster, J. Genschel, U. Curth, C. Urbanke, C. Kang, and R. Hilgenfeld, "A common core for binding single-stranded DNA: structural comparison of the single-stranded DNA-binding proteins (SSB) from E. coli and human mitochondria," FEBS Letters, vol. 411, pp. 313-316, 1997.

[8] R. L. Flynn and L. Zou, "Oligonucleotide/oligosaccharide-binding fold proteins: a growing family of genome guardians," Critical Reviews in Biochemistry and Molecular Biology, vol. 45, no. 4, pp. 266-275, 2010.

[9] K. Chan, Y. Lee, C. Wang, H. Huang, and Y. Sun, "Single-Stranded DNA-Binding Protein Complex from Helicobacter pylori Suggests an ssDNA-Binding Surface," Journal of Molecular Biology, vol. 388, no. 3, pp. 508-519, 2009.

[10] D. L. Theobald, R. M. Mitton-Fry, and D. S. Wuttke, "Nucleic acid recognition by OB-fold proteins," Annual Review of Biophysics and Biomolecular Structure, vol. 32, pp. 115-133, 2003.

[11] S. Raghunathan, A. G. Kozlov, T. M. Lohman, and G. Waksman, "Structure of the DNA binding domain of E. coli SSB bound to 
ssDNA," Nature Structural Biology, vol. 7, no. 8, pp. 648-652, 2000.

[12] A. G. Murzin, "OB(oligonucleotide/oligosaccharide binding)fold: common structural and functional solution for nonhomologous sequences," EMBO Journal, vol. 12, no. 3, pp. 861$867,1993$.

[13] N. P. George, K. V. Ngo, S. Chitteni-Pattu et al., "Structure and cellular dynamics of deinococcus radiodurans single-stranded DNA (ssDNA)-binding protein (SSB)-DNA complexes," The Journal of Biological Chemistry, vol. 287, no. 26, pp. 22123-22132, 2012.

[14] P. Filipkowski and J. Kur, "Identification and properties of the Deinococcus grandis and Deinococcus proteolyticus single-stranded DNA binding proteins (SSB)," Acta Biochimica Polonica, vol. 54, no. 1, pp. 79-87, 2007.

[15] P. Filipkowski, A. Duraj-Thatte, and J. Kur, "Identification, cloning, expression, and characterization of a highly thermostable single-stranded-DNA-binding protein (SSB) from Deinococcus murrayi," Protein Expression and Purification, vol. 53, no. 1, pp. 201-208, 2007.

[16] P. Filipkowski, M. Koziatek, and J. Kur, "A highly thermostable, homodimeric single-stranded DNA-binding protein from Deinococcus radiopugnans," Extremophiles, vol. 10, no. 6, pp. 607-614, 2006

[17] P. Filipkowski, A. Duraj-Thatte, and J. Kur, "Novel thermostable single-stranded DNA-binding protein (SSB) from Deinococcus geothermalis," Archives of Microbiology, vol. 186, no. 2, pp. 129$137,2006$.

[18] G. Witte, C. Urbanke, and U. Curth, "Single-stranded DNAbinding protein of Deinococcus radiodurans: a biophysical characterization," Nucleic Acids Research, vol. 33, no. 5, pp. 16621670, 2005.

[19] D. A. Bernstein, J. M. Eggingtont, M. P. Killoran, A. M. Misic, M. M. Cox, and J. L. Keck, "Crystal structure of the Deinococcus radiodurans single-stranded DNA-binding protein suggests a mechanism for coping with DNA damage," Proceedings of the National Academy of Sciences of the United States of America, vol. 101, no. 23, pp. 8575-8580, 2004.

[20] S. Dabrowski, M. Olszewski, R. Piatek, A. BrillowskaDabrowska, G. Konopa, and J. Kur, "Identification and characterization of single-stranded-DNA-binding proteins from Thermus thermophilus and Thermus aquaticus-new arrangement of binding domains," Microbiology, vol. 148, no. 10, pp. 3307-3315, 2002.

[21] R. Gamsjaeger, R. Kariawasam, C. Touma, A. H. Kwan, M. F. White, and L. Cubeddu, "Backbone and side-chain ${ }^{1} \mathrm{H},{ }^{13} \mathrm{C}$ and ${ }^{15} \mathrm{~N}$ resonance assignments of the $\mathrm{OB}$ domain of the single stranded DNA binding protein from Sulfolobus solfataricus and chemical shift mapping of the DNA-binding interface," Biomolecular NMR Assignments, 2013.

[22] M. L. Rolfsmeier and C. A. Haseltine, "The single-stranded DNA binding protein of Sulfolobus solfataricus acts in the presynaptic step of homologous recombination," Journal of Molecular Biology, vol. 397, no. 1, pp. 31-45, 2010.

[23] I. D. Kerr, R. I. M. Wadsworth, L. Cubeddu, W. Blankenfeldt, J. H. Naismith, and M. F. White, "Insights into ssDNA recognition by the OB fold from a structural and thermodynamic study of Sulfolobus SSB protein," EMBO Journal, vol. 22, no. 11, pp. 25612570, 2003.
[24] C. A. Haseltine and S. C. Kowalczykowski, "A distinctive singlestranded DNA-binding protein from the Archaeon Sulfolobus solfataricus," Molecular Microbiology, vol. 43, no. 6, pp. 1505$1515,2002$.

[25] R. I. M. Wadsworth and M. F. White, "Identification and properties of the crenarchaeal single-stranded DNA binding protein from Sulfolobus solfataricus," Nucleic Acids Research, vol. 29, no. 4, pp. 914-920, 2001.

[26] S. Sugiman-Marangos and M. S. Junop, “The structure of DdrB from Deinococcus: a new fold for single-stranded DNA binding proteins," Nucleic Acids Research, vol. 38, no. 10, Article ID gkq036, pp. 3432-3440, 2010.

[27] H. Ghalei, H. V. Moeller, D. Eppers et al., "Entrapment of DNA in an intersubunit tunnel system of a single-stranded DNAbinding protein," Nucleic Acids Research, vol. 42, no. 10, pp. 6698-6708, 2014.

[28] S. Paytubi, S. A. McMahon, S. Graham et al., "Displacement of the canonical single-stranded DNA-binding protein in the thermoproteales," Proceedings of the National Academy of Sciences of the United States of America, vol. 109, no. 7, pp. E398-E405, 2012.

[29] T. H. Dickey, S. E. Altschuler, and D. S. Wuttke, "Singlestranded DNA-binding proteins: multiple domains for multiple functions," Structure, vol. 21, no. 7, pp. 1074-1084, 2013.

[30] D. Vujaklija and B. Macek, "Detecting posttranslational modifications of bacterial SSB proteins," Methods in Molecular Biology, vol. 922, pp. 205-218, 2012.

[31] I. Mijakovic, D. Petranovic, B. Macek et al., "Bacterial singlestranded DNA-binding proteins are phosphorylated on tyrosine," Nucleic Acids Research, vol. 34, no. 5, pp. 1588-1596, 2006.

[32] M. Olszewski, A. Grot, M. Wojciechowski, M. Nowak, M. Mickiewicz, and J. Kur, "Characterization of exceptionally thermostable single-stranded DNA-binding proteins from Thermotoga maritima and Thermotoga neapolitana," BMC Microbiology, vol. 10, article 260, 2010.

[33] U. Curth, J. Genschel, C. Urbanke, and J. Greipel, "In vitro and in vivo function of the C-terminus of Escherichia coil singlestranded DNA binding protein," Nucleic Acids Research, vol. 24, no. 14 , pp. 2706-2711, 1996.

[34] G. Witte, C. Urbanke, and U. Curth, "DNA polymerase III chi subunit ties single-stranded DNA binding protein to the bacterial replication machinery," Nucleic Acids Research, vol. 31, pp. 4434-4440, 2003.

[35] D. Shishmarev, Y. Wang, C. E. Mason et al., "Otting, Intramolecular binding mode of the $\mathrm{C}$-terminus of Escherichia coli singlestranded DNA binding protein determined by nuclear magnetic resonance spectroscopy," Nucleic Acids Research, vol. 42, pp. 2750-2757, 2014.

[36] A. G. Kozlov, M. M. Cox, and T. M. Lohman, "Regulation of single-stranded DNA binding by the $\mathrm{C}$ termini of Escherichia coli single-stranded DNA-binding (SSB) protein," Journal of Biological Chemistry, vol. 285, no. 22, pp. 17246-17252, 2010.

[37] M. Nowak, M. Olszewski, M. Spibida, and J. Kur, "Characterization of single-stranded DNA-binding proteins from the psychrophilic bacteria Desulfotalea psychrophila, Flavobacterium psychrophilum, Psychrobacter arcticus, Psychrobacter cryohalolentis, Psychromonas ingrahamii, Psychroflexus torquis, and Photobacterium profundum," BMC Microbiology, vol. 14, article 91, 2014. 
[38] T. Paradzik, N. Ivic, Z. Filic et al., "Structure-function relationships of two paralogous single-stranded DNA-binding proteins from Streptomyces coelicolor: implication of SsbB in chromosome segregation during sporulation," Nucleic Acids Research, vol. 41, no. 6, pp. 3659-3672, 2013.

[39] S. Jain, M. Zweig, E. Peeters et al., "Characterization of the single stranded DNA binding protein SsbB encoded in the gonoccocal genetic island," PLoS ONE, vol. 7, no. 4, Article ID e35285, 2012.

[40] Y. H. Huang and C. Y. Huang, "Characterization of a singlestranded DNA-binding protein from Klebsiella pneumoniae: mutation at either Arg73 or Ser76 causes a less cooperative complex on DNA," Genes to Cells, vol. 17, no. 2, pp. 146-157, 2012.

[41] E. Antony, E. A. Weiland, S. Korolev, and T. M. Lohman, "Plasmodium falciparum SSB tetramer wraps single-stranded DNA with similar topology but opposite polarity to E. Coli SSB," Journal of Molecular Biology, vol. 420, no. 4-5, pp. 269-283, 2012.

[42] H. Jan, Y. L. Lee, and C. Y. Huang, "Characterization of a singlestranded DNA-binding protein from pseudomonas aeruginosa PAO1," Protein Journal, vol. 30, no. 1, pp. 20-26, 2011.

[43] Y.-H. Huang, Y.-L. Lee, and C.-Y. Huang, "Characterization of a single-stranded DNA binding protein from Salmonella enterica serovar typhimurium LT2," Protein Journal, vol. 30, no. 2, pp. 102-108, 2011.

[44] S. K. Bharti, K. Rex, P. Sreedhar, N. Krishnan, and U. Varshney, "Chimeras of Escherichia coli and Mycobacterium tuberculosis single-stranded DNA binding proteins: characterization and function in Escherichia coli," PLoS ONE, vol. 6, no. 12, Article ID e27216, 2011.

[45] M. T. Oliveira and L. S. Kaguni, "Functional roles of the Nand C-terminal regions of the human mitochondrial singlestranded DNA-binding protein," PLoS ONE, vol. 5, no. 10, Article ID e15379, 2010.

[46] A. G. Kozlov, J. M. Eggington, M. M. Cox, and T. M. Lohman, "Binding of the dimeric Deinococcus radiodurans single-stranded DNA binding protein to single-stranded DNA," Biochemistry, vol. 49, no. 38, pp. 8266-8275, 2010.

[47] T. M. Lohman and M. E. Ferrari, "Escherichia coli singlestranded DNA-binding protein: multiple DNA-binding modes and cooperatives," Annual Review of Biochemistry, vol. 63, pp. 527-570, 1994.

[48] R. Zhou, A. G. Kozlov, R. Roy et al., "SSB functions as a sliding platform that migrates on DNA via reptation," Cell, vol. 146, pp. 222-232, 2011.

[49] R. Roy, A. G. Kozlov, T. M. Lohman, and T. Ha, "SSB protein diffusion on single-stranded DNA stimulates RecA filament formation," Nature, vol. 461, pp. 1092-1097, 2009.

[50] R. Roy, A. G. Kozlov, T. M. Lohman, and T. Ha, "Dynamic structural rearrangements between DNA binding modes of $\mathrm{E}$. coli SSB protein," Journal of Molecular Biology, vol. 369, no. 5, pp. 1244-1257, 2007.

[51] T. J. Kelly, P. Simancek, and G. S. Brush, "Identification and characterization of a single-stranded DNA-binding protein from the archaeon Methanococcus jannaschii," Proceedings of the National Academy of Sciences of the United States of America, vol. 95, no. 25, pp. 14634-14639, 1998.

[52] C. Y. Huang, Determination of the Binding Site-Size of the Protein-DNA Complex by Use of the Electrophoretic Mobility Shift Assay, InTech Press, Rijeka, Croatia, 2012.
[53] A. Kornberg, "Ten commandments of enzymology, amended," Trends in Biochemical Sciences, vol. 28, no. 10, pp. 515-517, 2003.

[54] W. Zhang, X. Lü, and J. Shen, "EMSA and single-molecule force spectroscopy study of interactions between bacillus subtilis single-stranded DNA-binding protein and single-stranded DNA," Langmuir, vol. 27, no. 24, pp. 15008-15015, 2011.

[55] M. Ostermeier and S. J. Benkovic, "Evolution of protein function by domain swapping," Advances in Protein Chemistry, vol. 55, pp. 29-77, 2000.

[56] W. F. Peng and C. Y. Huang, "Allantoinase and dihydroorotase binding and inhibition by flavonols and the substrates of cyclic amidohydrolases," Biochimie, vol. 101, pp. 113-122, 2014.

[57] Y. H. Huang, M. J. Lin, and C. Y. Huang, "DnaT is a singlestranded DNA binding protein," Genes to Cells, vol. 18, no. 11, pp. 1007-1019, 2013.

[58] Y. Y. Ho, Y. H. Huang, and C. Y. Huang, "Chemical rescue of the post-translationally carboxylated lysine mutant of allantoinase and dihydroorotase by metal ions and short-chain carboxylic acids," Amino Acids, vol. 44, no. 4, pp. 1181-1191, 2013.

[59] Y. H. Huang, Y. Lo, W. Huang, and C. Y. Huang, "Crystal structure and DNA-binding mode of Klebsiella pneumoniae primosomal PriB protein," Genes to Cells, vol. 17, no. 10, pp. 837849, 2012.

[60] C. Y. Huang, C. Y. Hsu, Y. Sun, H. Wu, and C. Hsiao, "Complexed crystal structure of replication restart primosome protein PriB reveals a novel single-stranded DNA-binding mode," Nucleic Acids Research, vol. 34, no. 14, pp. 3878-3886, 2006.

[61] T. Kinebuchi, H. Shindo, H. Nagai, N. Shimamoto, and M. Shimizu, "Functional domains of Escherichia coli singlestranded DNA binding protein as assessed by analyses of the deletion mutants," Biochemistry, vol. 36, no. 22, pp. 6732-6738, 1997.

[62] N. Shimamoto, N. Ikushima, H. Utiyama, H. Tachibana, and K. Horie, "Specific and cooperative binding of E. coli singlestranded DNA binding protein to mRNA," Nucleic Acids Research, vol. 15, no. 13, pp. 5241-5250, 1987.

[63] H. Lin and C. Y. Huang, "Characterization of flavonol inhibition of DnaB helicase: Real-time monitoring, structural modeling, and proposed mechanism," Journal of Biomedicine and Biotechnology, vol. 2012, Article ID 735368, 2012.

[64] Y. H. Huang, H. H. Lin, and C. Y. Huang, "A single residue determines the cooperative binding property of a primosomal DNA replication protein, PriB, to single-stranded DNA," Bioscience, Biotechnology and Biochemistry, vol. 76, no. 6, pp. 11101115, 2012.

[65] H. C. Hsieh and C. Y. Huang, "Identification of a novel protein, PriB, in Klebsiella pneumoniae," Biochemical and Biophysical Research Communications, vol. 404, no. 1, pp. 546-551, 2011.

[66] J. H. Liu, T. W. Chang, C. Y. Huang et al., "Crystal structure of PriB, a primosomal DNA replication protein of Escherichia coli," The Journal of Biological Chemistry, vol. 279, no. 48, pp. 5046550471, 2004.

[67] Y. H. Huang and C. Y. Huang, "The N-terminal domain of DnaT, a primosomal DNA replication protein, is crucial for PriB binding and self-trimerization," Biochemical and Biophysical Research Communications, vol. 442, pp. 147-152, 2013.

[68] M. A. Larkin, G. Blackshields, N. P. Brown et al., "Clustal W and Clustal X version 2.0,” Bioinformatics, vol. 23, no. 21, pp. 29472948, 2007. 
[69] S. N. Savvides, S. Raghunathan, K. Fütterer, A. G. Kozlov, T. M. Lohman, and G. Waksman, "The C-terminal domain of fulllength E. coli SSB is disordered even when bound to DNA," Protein Science, vol. 13, no. 7, pp. 1942-1947, 2004.

[70] C.-C. Chen, J.-K. Hwang, and J.-M. Yang, "(PS)2-v2: templatebased protein structure prediction server," BMC Bioinformatics, vol. 10, article 366, 2009.

[71] C. Chen, J. Hwang, and J. Yang, "(PS)2: protein structure prediction server," Nucleic Acids Research, vol. 34, pp. W152-W157, 2006.

[72] K. Bush and J. F. Fisher, "Epidemiological expansion, structural studies, and clinical challenges of new $\beta$-lactamases from gramnegative bacteria," Annual Review of Microbiology, vol. 65, pp. 455-478, 2011.

[73] K. K. Kumarasamy, M. A. Toleman, T. R. Walsh et al., "Emergence of a new antibiotic resistance mechanism in India, Pakistan, and the UK: a molecular, biological, and epidemiological study," The Lancet Infectious Diseases, vol. 10, no. 9, pp. 597-602, 2010.

[74] K. Bush and M. J. MacIelag, "New $\beta$-lactam antibiotics and $\beta$ lactamase inhibitors," Expert Opinion on Therapeutic Patents, vol. 20, no. 10, pp. 1277-1293, 2010.

[75] K. Bush, "Alarming $\beta$-lactamase-mediated resistance in multidrug-resistant Enterobacteriaceae," Current Opinion in Microbiology, vol. 13, no. 5, pp. 558-564, 2010.

[76] A. Srivastava, M. Talaue, S. Liu et al., "New target for inhibition of bacterial RNA polymerase: "switch region'”, Current Opinion in Microbiology, vol. 14, no. 5, pp. 532-543, 2011.

[77] K. Chono, K. Katsumata, T. Kontani et al., "ASP2151, a novel helicase-primase inhibitor, possesses antiviral activity against varicella-zoster virus and herpes simplex virus types 1 and 2," Journal of Antimicrobial Chemotherapy, vol. 65, no. 8, pp. 1733$1741,2010$.

[78] M. T. Black and K. Coleman, "New inhibitors of bacterial topoisomerase GyrA/ParC subunits," Current Opinion in Investigational Drugs, vol. 10, no. 8, pp. 804-810, 2009.

[79] A. H. Marceau, D. A. Bernstein, B. W. Walsh, W. Shapiro, L. A. Simmons, and J. L. Keck, "Protein interactions in genome maintenance as novel antibacterial targets," PLOS ONE, vol. 8, no. 3, Article ID e58765, 2013.

[80] D. Lu, D. A. Bernstein, K. A. Satyshur, and J. L. Keck, "Smallmolecule tools for dissecting the roles of SSB/protein interactions in genome maintenance," Proceedings of the National Academy of Sciences of the United States of America, vol. 107, no. 2, pp. 633-638, 2010.

[81] I. Wong and T. M. Lohman, "A double-filter method for nitrocellulose-filter binding: application to protein-nucleic acid interactions," Proceedings of the National Academy of Sciences of the United States of America, vol. 90, no. 12, pp. 5428-5432, 1993.

[82] M. Mitas, J. Y. Chock, and M. Christy, “The binding-site sizes of Escherichia coli single-stranded-DNA-binding protein and mammalian replication protein $\mathrm{A}$ are 65 and $\geq 54$ nucleotides respectively," Biochemical Journal, vol. 324, no. 3, pp. 957-961, 1997.

[83] C. Urbanke, G. Witte, and U. Curth, "Sedimentation velocity method in the analytical ultracentrifuge for the study of proteinprotein interactions," Methods in Molecular Biology, vol. 305, pp. 101-114, 2005.
[84] S. Chrysogelos and J. Griffith, "Escherichia coli single-strand binding protein organizes single-stranded DNA in nucleosomelike units," Proceedings of the National Academy of Sciences of the United States of America, vol. 79, no. 19, pp. 5803-5807, 1982.

[85] J. R. Casas-Finet, K. R. Fischer, and R. L. Karpel, "Structural basis for the nucleic acid binding cooperativity of bacteriophage T4 gene 32 protein: the (Lys/Arg)3(Ser/Thr)2 (LAST) motif," Proceedings of the National Academy of Sciences of the United States of America, vol. 89, pp. 1050-1054, 1992.

[86] G. Witte, R. Fedorov, and U. Curth, "Biophysical analysis of Thermus aquaticus single-stranded DNA binding protein," Biophysical Journal, vol. 94, no. 6, pp. 2269-2279, 2008.

[87] R. Fedorov, G. Witte, C. Urbanke, D. J. Manstein, and U. Curth, "3D structure of Thermus aquaticus single-stranded DNA-binding protein gives insight into the functioning of SSB proteins," Nucleic Acids Research, vol. 34, no. 22, pp. 6708-6717, 2006.

[88] K. L. Tsai, C. Y. Huang, C. H. Chang, Y. J. Sun, W. J. Chuang, and C. D. Hsiao, "Crystal structure of the human FOXK1a-DNA complex and its implications on the diverse binding specificity of winged helix/forkhead proteins," Journal of Biological Chemistry, vol. 281, no. 25, pp. 17400-17409, 2006.

[89] A. H. Mao, N. Lyle, and R. V. Pappu, "Describing sequenceensemble relationships for intrinsically disordered proteins," Biochemical Journal, vol. 449, no. 2, pp. 307-318, 2013.

[90] R. Wetzel, "Physical chemistry of polyglutamine: Intriguing tales of a monotonous sequence," Journal of Molecular Biology, vol. 421, no. 4-5, pp. 466-490, 2012.

[91] H. T. Orr, "Polyglutamine neurodegeneration: expanded glutamines enhance native functions," Current Opinion in Genetics and Development, vol. 22, no. 3, pp. 251-255, 2012.

[92] M. Figiel, W. J. Szlachcic, P. M. Switonski, A. Gabka, and W. J. Krzyzosiak, "Mouse models of polyglutamine diseases: review and data table. Part I," Molecular Neurobiology, vol. 46, no. 2, pp. 393-429, 2012.

[93] J. Nasica-Labouze and N. Mousseau, "Kinetics of amyloid aggregation: a study of the GNNQQNY prion sequence," PLoS Computational Biology, vol. 8, no. 11, Article ID e1002782, 2012.

[94] J. Nasica-Labouze, M. Meli, P. Derreumaux, G. Colombo, and N. Mousseau, "A multiscale approach to characterize the early aggregation steps of the amyloid-forming peptide gnnqqny from the yeast prion sup-35," PLoS Computational Biology, vol. 7, no. 5, Article ID e1002051, 2011.

[95] J. R. Lewandowski, P. C. A. van der Wel, M. Rigney, N. Grigorieff, and R. G. Griffin, "Structural complexity of a composite amyloid fibril," Journal of the American Chemical Society, vol. 133, no. 37, pp. 14686-14698, 2011. 

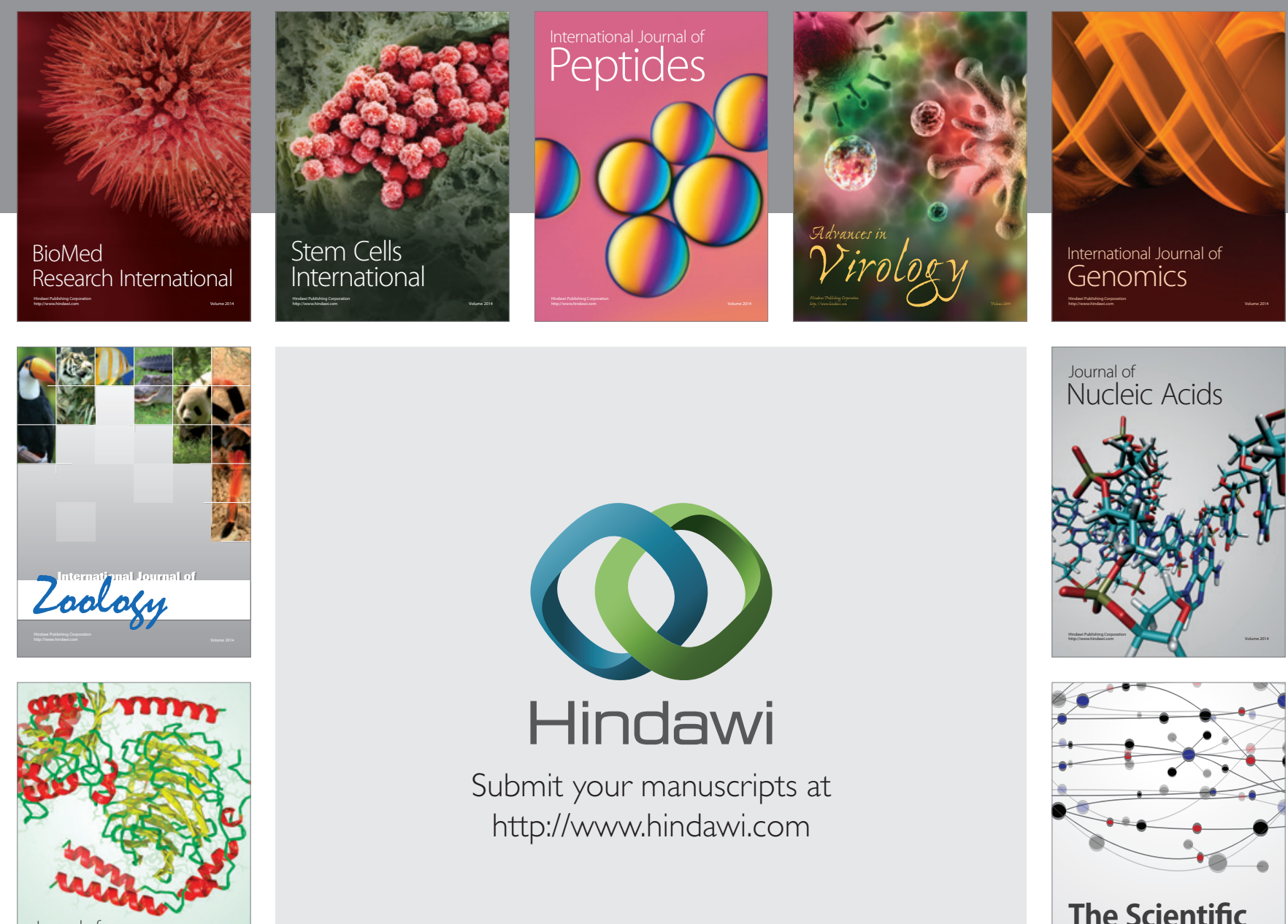

Submit your manuscripts at

http://www.hindawi.com

Journal of
Signal Transduction
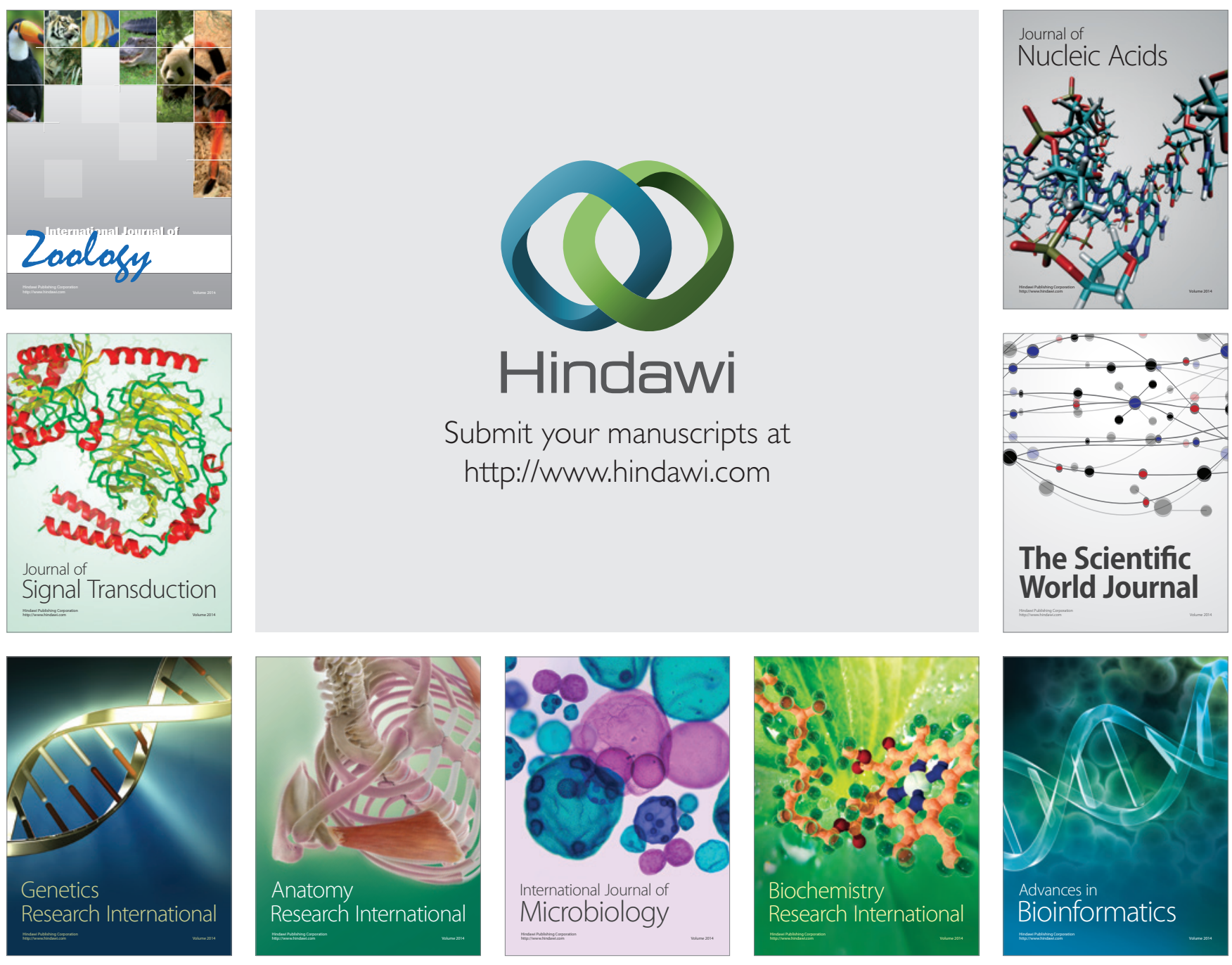

The Scientific World Journal
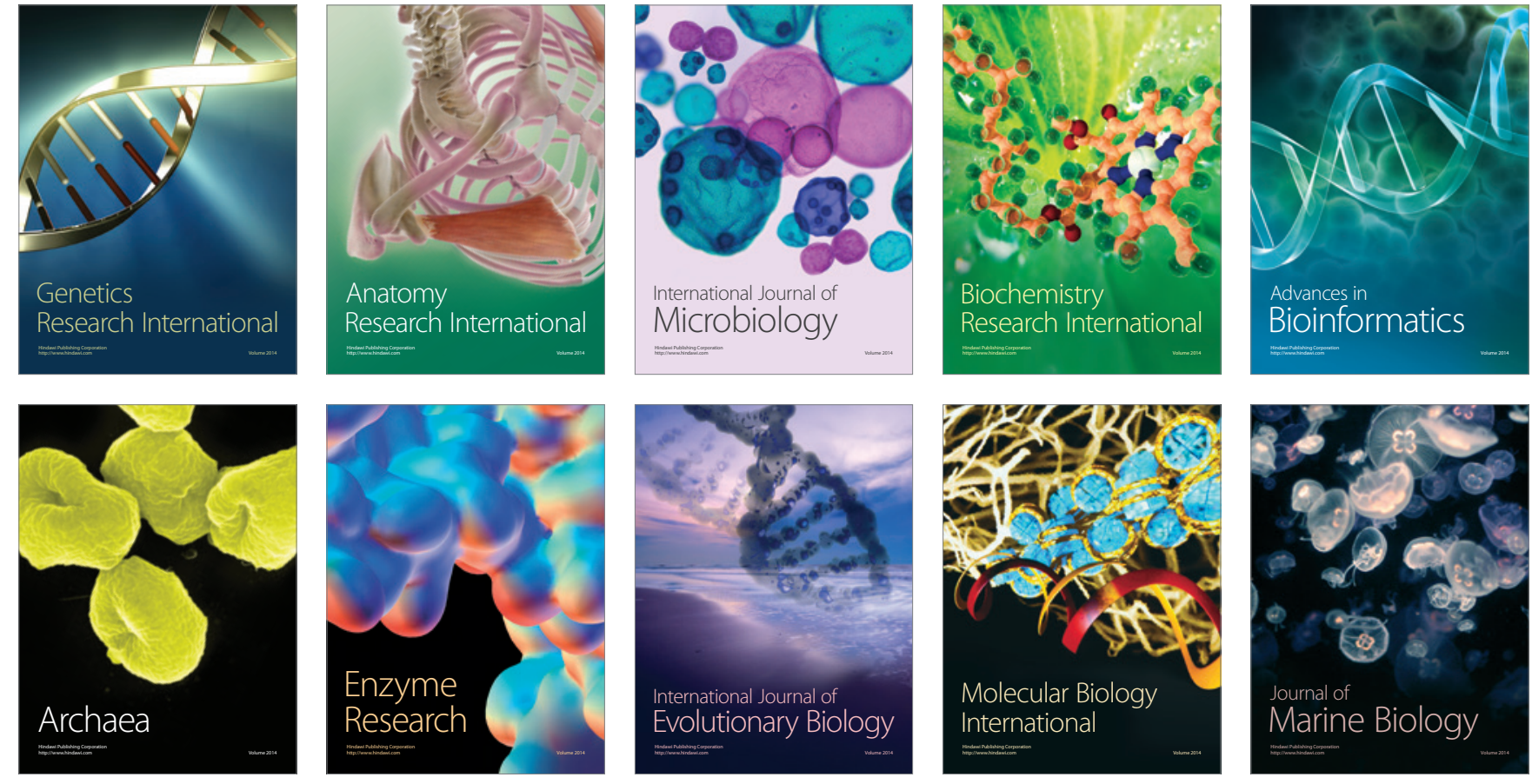